MATHEMATICS OF COMPUTATION

Volume 80, Number 276, October 2011, Pages 1887-1910

S 0025-5718(2011)02464-6

Article electronically published on February 4, 2011

\title{
APPROXIMATION OF THE EIGENVALUE PROBLEM FOR THE TIME HARMONIC MAXWELL SYSTEM BY CONTINUOUS LAGRANGE FINITE ELEMENTS
}

\author{
ANDREA BONITO AND JEAN-LUC GUERMOND
}

\begin{abstract}
We propose and analyze an approximation technique for the Maxwell eigenvalue problem using $\mathbf{H}^{1}$-conforming finite elements. The key idea consists of considering a mixed method controlling the divergence of the electric field in a fractional Sobolev space $H^{-\alpha}$ with $\alpha \in\left(\frac{1}{2}, 1\right)$. The method is shown to be convergent and spectrally correct.
\end{abstract}

\section{INTRODUCTION}

We consider the time-harmonic three-dimensional Maxwell equations with perfect conductor boundary conditions in a simply connected, bounded, Lipschitz domain $\Omega \subset \mathbb{R}^{d}, d=2,3$ :

$$
\begin{aligned}
& \nabla \times \mathbf{E}-i \omega \mu \mathbf{H}=0 \text { and } \nabla \times \mathbf{H}+i \omega \varepsilon \mathbf{E}=\mathbf{J} \quad \text { in } \Omega, \\
& \mathbf{E} \times \mathbf{n}=0 \text { and } \mathbf{H} \cdot \mathbf{n}=0 \text { on } \partial \Omega .
\end{aligned}
$$

The coefficients are assumed to be constant and, without loss of generality, we henceforth assume that $\mu \varepsilon=1$. Eliminating the magnetic field $\mathbf{H}$ from the above system, the electric field satisfies the following PDE system:

$$
\nabla \times \nabla \times \mathbf{E}-\omega^{2} \mathbf{E}=\mathbf{f} \text { and } \nabla \cdot \mathbf{E}=0, \quad \text { in } \Omega,
$$

where $\nabla \cdot \mathbf{f}=0$, naturally raising the question of the eigenvalue problem

$$
\nabla \times \nabla \times \mathbf{E}=\lambda \mathbf{E} \quad \text { and } \quad \nabla \cdot \mathbf{E}=0, \quad \text { and } \quad \mathbf{E} \times\left.\mathbf{n}\right|_{\partial \Omega}=0 .
$$

The objective of this paper is to propose and analyze an approximation technique for the eigenvalue problem (1.2) using $\mathcal{C}^{0}$-Lagrange finite elements. This task is quite challenging since it has been shown by Costabel in 18 that any $\mathbf{H}^{1}$ conforming approximation technique that induces uniform $L^{2}$-stability estimates both on the curl and the divergence of the approximate electric field cannot converge if $\Omega$ is non-smooth and non-convex.

Received by the editor October 1, 2009 and, in revised form, July 12, 2010.

2010 Mathematics Subject Classification. 65N25, 65F15, 35Q61.

Key words and phrases. Finite elements, Maxwell equations, eigenvalue, spectral approximation.

The first author was partially supported by the NSF grant DMS-0914977.

The second author was partially supported by Award No. KUS-C1-016-04, made by King Abdullah University of Science and Technology (KAUST).

The third author was partially supported by the NSF grant DMS-07138229.

(C)2011 American Mathematical Society Reverts to public domain 28 years from publication 
In the present paper we follow an idea developed by Costabel and Dauge [19] and Bramble et al. 6, 4] which consists of controlling the divergence of the approximate electric field in a space which is intermediate between $L^{2}(\Omega)$ and $H^{-1}(\Omega)$. This is done in 6, 4, by formulating the problem with different test and trial spaces and, finally, constructing a least-square approximation; the solution space is $\mathbf{L}^{2}(\Omega)$ and the components of the trial space are subspaces of $H^{1}(\Omega)$. In [19] this program is carried out by controlling the divergence of the electric field in a weighted $L^{2}$-space where the weight is a distance to the re-entrant corners of the domain to some appropriate power. The $L^{2}$-approximation theory of Bramble et al. is optimal for boundary value and eigenvalue problems. During the review process of the present work we have been informed of a recent result by A. Buffa, P. Ciarlet, and E. Jamelot [10] showing that a mixed form of the weighted $L^{2}$-stabilization technique is also spectrally correct.

The idea that is developed in the present paper is to stabilize the divergence of the electric field in the space $H^{-\alpha}(\Omega)$ with $\alpha \in\left(\frac{1}{2}, 1\right)$. The main result of the paper is Theorem 5.1, which hinges on the following key result (Lemma 3.1)

$$
c\|\mathbf{F}\|_{\mathbf{H}^{1-\alpha}(\Omega)} \leq\|\nabla \times \mathbf{F}\|_{\mathbf{L}^{2}(\Omega)}+\|\nabla \cdot \mathbf{F}\|_{\mathbf{H}^{-\alpha}(\Omega)},
$$

which holds true for all fields with zero tangent trace provided that $\Omega$ is simply connected. The proposed technique is characterized by the following novelties:

- The approximating finite element space is not required to contain gradients. As a consequence, the convergence of the eigenvalue problem is obtained without any restriction on the finite element space.

- The stabilization of the divergence is performed using meshsize-dependent bilinear forms but does not require any additional computations such as the distance to the corners of the domain. This is particularly relevant in three dimensions and for moving domains for which the computation needs to be performed at each step.

- No extra regularity of the type $\|p\|_{H^{\frac{3}{2}+\epsilon}} \leq c\|\Delta p\|_{L^{2}}$ leading to restrictions on the domain interior angles of the domain is assumed; see e.g. 37, 21. Although the $H^{\frac{3}{2}+\epsilon}$-regularity is achieved in most Lipschitz domains, it may become quite restrictive when the permeability and permittivity fields are discontinuous.

The paper is organized as follows. Notation and preliminary technicalities are introduced in $\$ 2$ The approximation technique based on the control of the divergence in $H^{-\alpha}(\Omega)$ is introduced and analyzed in 93 . The method is quite awkward since computing an $H^{-\alpha}$-norm with $\alpha \in\left(\frac{1}{2}, 1\right)$ and with Lagrange finite elements requires a hierarchical decomposition of the approximation space which may not always be available. To circumvent this difficulty and make the method more practical we introduce a relaxed version thereof in 4 . The idea is to modify the formulation to account for the fact that $H^{-\alpha}(\Omega)$ is an interpolation space between $L^{2}(\Omega)$ and $H^{-1}(\Omega)$. Although the methods introduced in $\$ 3$ and 94 are convergent for the boundary value problem (1.1) with $\omega=0$, they do not correctly solve the eigenvalue problem (1.2) due to a consistency default appearing when the righthand side is not exactly solenoidal. A mixed method that remedies the consistency problem and is easy to implement is introduced and analyzed in 95 . The purpose of Sections $\$ 3$ and 4 is to guide the reader through the genesis of the method. Finally, numerical tests illustrating the method described in $\$ 5$ are reported in $\$ 6$. 


\section{NOTATION AND PRELIMINARIES}

2.1. Functional spaces. Let $\Omega$ be a bounded, simply connected, Lipschitz domain in $\mathbb{R}^{d}, d=2,3$. The unit outer normal vector at the boundary $\partial \Omega$ is denoted $\mathbf{n}$. The scalar product in $L^{2}(\Omega)$ and $\mathbf{L}^{2}(\Omega)$ is denoted $(\cdot, \cdot)$; no notational distinction is made between the scalar-valued and vector-valued scalar product.

For $0<s<1$, the space $H^{s}(\Omega):=\left[L^{2}(\Omega), H^{1}(\Omega)\right]_{s}$ is defined by the real method of interpolation between $L^{2}(\Omega)$ and $H^{1}(\Omega)$, i.e., the so-called K-method of [36; see also [35] or [5, Appendix A]. We interpolate between $H^{1}(\Omega)$ and $H^{2}(\Omega)$ if $1<s<2$. We denote $H_{0}^{1}(\Omega)$ the closure of $C_{0}^{\infty}(\Omega)$ in $H^{1}(\Omega)$ and we set $H_{0}^{s}(\Omega):=\left[L^{2}(\Omega), H_{0}^{1}(\Omega)\right]_{s}$. (This definition is slightly different from what is usually done; the only differences occurs at $s=\frac{1}{2}$. Hereafter, what we denote by $H_{0}^{\frac{1}{2}}(\Omega)$ is usually denoted by $H_{00}^{\frac{1}{2}}(\Omega)$ elsewhere.) Let us recall that the spaces $H_{0}^{s}(\Omega)$ and $H^{s}(\Omega)$ coincide for $s \in\left[0, \frac{1}{2}\right.$ ) and their norms are equivalent, (see e.g. [35, Thm 11.1] or [27, Cor 1.4.4.5]). Recall also that $\mathcal{C}_{0}^{\infty}(\Omega)$ is dense in $H_{0}^{s}(\Omega)$ for $s \in[0,1]$. The space $H^{-s}(\Omega)$ is defined by duality with $H_{0}^{s}(\Omega)$ for $0 \leq s \leq 1$, i.e.,

$$
\|v\|_{H^{-s}}=\sup _{0 \neq w \in H_{0}^{s}(\Omega)} \frac{(v, w)}{\|w\|_{H^{s}}} .
$$

It is a standard result that $H^{-s}(\Omega)=\left[H^{-1}(\Omega), L^{2}(\Omega)\right]_{s}$, i.e., $\left[L^{2}(\Omega), H^{-1}(\Omega)\right]_{s}=$ $\left[H_{0}^{1}(\Omega), L^{2}(\Omega)\right]_{s}^{\prime}$, and the $H^{-s}$-scalar product can be written as

$$
\langle., .\rangle_{-s}=\left\langle\cdot,\left(-\Delta_{D}\right)^{-s} \cdot\right\rangle_{-s, s},
$$

where $\langle\cdot, \cdot\rangle_{-s, s}$ denotes the $H^{-s}-H^{s}$ pairing and $\Delta_{D}$ is the Laplace operator with zero Dirichlet boundary condition. The above definitions are naturally extended to the vector-valued Sobolev spaces $\mathbf{H}^{s}(\Omega)$ and $\mathbf{H}_{0}^{s}(\Omega)$.

We shall also use the following spaces equipped with their natural norms:

$$
\begin{aligned}
& \mathbf{H}(\operatorname{curl}, \Omega)=\left\{\mathbf{F} \in \mathbf{L}^{2}(\Omega) ; \nabla \times \mathbf{F} \in \mathbf{L}^{2}(\Omega)\right\}, \\
& \mathbf{H}(\operatorname{div}, \Omega)=\left\{\mathbf{F} \in \mathbf{L}^{2}(\Omega) ; \nabla \cdot \mathbf{F} \in L^{2}(\Omega)\right\}, \\
& \mathbf{H}_{0}(\operatorname{div}, \Omega)=\left\{\mathbf{F} \in \mathbf{H}(\operatorname{div}, \Omega) ;\left.\mathbf{F} \cdot \mathbf{n}\right|_{\partial \Omega}=0\right\}, \\
& \mathbf{H}_{0}(\operatorname{curl}, \Omega)=\left\{\mathbf{F} \in \mathbf{H}(\operatorname{curl}, \Omega) ; \mathbf{F} \times\left.\mathbf{n}\right|_{\partial \Omega}=0\right\}, \\
& \mathbf{H}(\operatorname{div}=0, \Omega)=\{\mathbf{F} \in \mathbf{H}(\operatorname{div}, \Omega) ; \nabla \cdot \mathbf{F}=0\}, \\
& \mathbf{X}_{0}:=\mathbf{H}_{0}(\operatorname{curl}, \Omega) \cap \mathbf{H}(\operatorname{div}=0, \Omega) .
\end{aligned}
$$

Henceforth, $c$ is a generic constant that does not depend on small parameters like the mesh size $h$ or the mollifying parameter $\epsilon$. The value of $c$ may change at each occurrence.

We recall the following regularity result for the Poisson problem.

Theorem 2.1 (Poisson Problem). Let $\alpha \in\left(\frac{1}{2}, 1\right]$. There is $c>0$ so that for all $f \in H^{-\alpha}(\Omega)$, there is a unique $p \in H^{2-\alpha}(\Omega) \cap H_{0}^{1}(\Omega)$ satisfying $\Delta p=f$ and $\|p\|_{H^{2-\alpha}} \leq c\|f\|_{H^{-\alpha}}$.

Proof. See Theorem 0.5 in [30].

The following Lemma will also be used repeatedly.

Lemma $2.1\left(\mathbf{H}^{\frac{1}{2}}(\Omega)\right.$ Estimate). There is a constant $c$ so that

$$
c\|\mathbf{v}\|_{\mathbf{H}^{\frac{1}{2}}} \leq\|\nabla \times \mathbf{v}\|_{\mathbf{L}^{2}}+\|\nabla \cdot \mathbf{v}\|_{\mathbf{L}^{2}}, \quad \mathbf{v} \in \mathbf{H}(\operatorname{div}, \Omega) \cap \mathbf{H}_{0}(\operatorname{curl}, \Omega) .
$$


Proof. Use Theorem 2 in [17] and the so-called Petree-Tartar Lemma (see e.g. 24, Lemma A.38] together with the fact that $\Omega$ is simply connected.

Remark $2.1\left(\mathbf{H}^{\frac{1}{2}}(\Omega)\right.$ Estimate). Actually, Theorem 2 in 17 asserts that both $\mathbf{H}(\operatorname{div}, \Omega) \cap \mathbf{H}_{0}(\operatorname{curl}, \Omega)$ and $\mathbf{H}_{0}(\operatorname{div}, \Omega) \cap \mathbf{H}(\operatorname{curl}, \Omega)$ are continuously embedded in $\mathbf{H}^{\frac{1}{2}}(\Omega)$. Lemma 2.1 is a specialization of this result when $\Omega$ is simply connected.

2.2. The eigenvalue problem. To reformulate the eigenvalue problem (1.2) in an appropriate functional setting we define the operator

$$
A: \mathbf{L}^{2}(\Omega) \rightarrow \mathbf{X}_{0}: \mathbf{E} \mapsto A \mathbf{E},
$$

where $A \mathbf{E}$ is the unique element in $\mathbf{X}_{0}$ satisfying

$$
(\nabla \times A \mathbf{E}, \nabla \times \mathbf{F})=(\mathbf{E}, \mathbf{F}), \quad \forall \mathbf{F} \in \mathbf{X}_{0} .
$$

The existence and the uniqueness of $A \mathbf{E}$ is a simple consequence of the Lax-Milgram Lemma. The definition of $A \mathbf{E}$ implies that there is $p \in H_{0}^{1}(\Omega)$ so that $\nabla \times \nabla \times A \mathbf{E}+$ $\nabla p=\mathbf{E}$; as a result, $A \mathbf{E}$ solves the following boundary value problem

$$
\nabla \times \nabla \times A \mathbf{E}+\nabla p=\mathbf{E}, \quad \nabla \cdot A \mathbf{E}=0, \quad A \mathbf{E} \times\left.\mathbf{n}\right|_{\partial \Omega}=0,\left.\quad p\right|_{\partial \Omega}=0 .
$$

Note, in particular, that the lagrange multiplier $p$ satisfies $\Delta p=\nabla \cdot \mathbf{E}, p \in H_{0}^{1}(\Omega)$, and

$$
\|\nabla p\|_{\mathbf{L}^{2}} \leq\|\nabla \cdot \mathbf{E}\|_{H^{-1}}
$$

The eigenvalue problem (1.2) is re-interpreted as follows:

Lemma 2.2. Let $\mathbf{E} \in \mathbf{H}_{0}$ (curl, $\left.\Omega\right) \backslash\{0\}$ and $\lambda \in \mathbb{R} \backslash\{0\}$. Then, $(\mathbf{E}, \lambda)$ is an eigenpair for (1.2) if and only if $(\mathbf{E}, 1 / \lambda)$ is an eigenpair for the operator $A$.

Proof. (i) Assume that $(\mathbf{E}, \lambda)$ is an eigenpair for (1.2). It immediately follows from (1.2) that $\mathbf{E} \in \mathbf{X}_{0}$. Since $\lambda \neq 0$, (1.2) also implies that $\left(\nabla \times\left(\lambda^{-1} \mathbf{E}\right), \nabla \times \mathbf{F}\right)=(\mathbf{E}, \mathbf{F})$ for all $\mathbf{F} \in \mathbf{X}_{0}$, which in turns means that $A \mathbf{E}=\lambda^{-1} \mathbf{E}$.

(ii) Assume that $(\mathbf{E}, 1 / \lambda)$ is an eigenpair for the operator $A$, i.e., $\lambda A \mathbf{E}=\mathbf{E}$. Definition (2.3) implies that there exists $p \in H_{0}^{1}(\Omega)$ so that $\nabla \times \nabla \times A \mathbf{E}+\nabla p=\mathbf{E}$. Since $\mathbf{E}=\lambda A \mathbf{E} \in \mathbf{X}_{0}$, we have $0=\nabla \cdot \mathbf{E}=\Delta p$, which implies $p=0$. As a result $\nabla \times \nabla \times \mathbf{E}=\lambda \mathbf{E}$.

Remark 2.2 (Non-zero Eigenvalues). The hypothesis $\lambda \neq 0$ in Lemma2.2 is justified by the fact that the eigenvalues of (1.2) are positive (cf. Lemma 2.1). Note also that $A$ is not injective, i.e., 0 is an eigenvalue of $A$ and $\nabla H_{0}^{1}(\Omega)$ is the associated eigenspace.

The objective of this paper is to describe and analyze an approximation technique for computing the spectrum of $A$ using Lagrange finite elements. Since the operator $A$ is self-adjoint and Lemma 2.1 implies that $A$ is also compact, we conclude this section by stating a result proved in 39 regarding the approximation eigenvalue problems for compact self-adjoint operators.

Let $X$ be a Hilbert space with inner product $(., .)_{X}$ and norm $\|\cdot\|_{X}$. Let $\Theta=$ $\left\{h_{n} ; n \in \mathbb{N}\right\}$ be a discrete subset of $\mathbb{R}$ such that $h_{n} \rightarrow 0$ as $n \rightarrow \infty$. A sequence of operators $\mathcal{A}=\left\{A_{h}: X \rightarrow X ; h \in \Theta\right\}$ is said to be collectively compact if for each bounded set $U \subset X$, the image set

$$
\mathcal{A} U=\left\{A_{h} \mathbf{F} ; \mathbf{F} \in U, A_{h} \in \mathcal{A}\right\}
$$


is relatively compact in $X$. We say that the sequence $\mathcal{A}$ converges pointwise to $A$ if for all $x \in X, A_{h} x \rightarrow A x$ in $X$ as $h \rightarrow 0$.

Theorem 2.2 (Spectral Convergence [39] and [2]). Let $X$ be a Hilbert space. Assume that the set $\mathcal{A}=\left\{A_{h}: X \rightarrow X ; h \in \Theta\right\}$ of linear self-adjoint operators in $X$ is collectively compact. Assume furthermore that there exists a self-adjoint and compact operator $A$ in $X$ such that $\mathcal{A}$ converges pointwise to $A$. Let $\mu$ be an eigenvalue of $A$ of multiplicity $m$ and denote by $\left\{\phi_{j}\right\}_{j=1}^{m}$ a set of associated orthonormal eigenvectors.

(i) For any $\epsilon>0$ such that the disk $B(\mu, \epsilon)$ of radius $\epsilon$ and center $\mu$ contains no other eigenvalues of $A$, there exists $h_{\epsilon}$ such that for all $h<h_{\epsilon}, A_{h}$ has exactly $m$ eigenvalues (repeated according to their multiplicity) in $B(\mu, \epsilon)$.

(ii) For $h<h_{\epsilon}$, if we denote by $\mu_{h, j}, j-1, \ldots, m$, the set of the eigenvalues of $A_{h}$ in $B(\mu, \epsilon)$, then for all $j=1, \ldots, m$, there exists a positive constant $c$ such that

$$
c\left|\mu-\mu_{h, j}\right| \leq \sum_{j, l=1}^{m}\left|\left(\left(A-A_{h}\right) \phi_{j}, \phi_{l}\right)_{X}\right|+\sum_{j=1}^{m}\left\|\left(A-A_{h}\right) \phi_{j}\right\|_{X}^{2} .
$$

2.3. Mollification. We gather in this section some results concerning regularization by mollification that will be used repeatedly in the rest of the paper.

Proposition 2.1 (Stability Estimate). Given $\mathbf{E} \in \mathbf{L}^{2}(\Omega)$, the solution AE to (2.3) satisfies the following regularity property:

$$
\|A \mathbf{E}\|_{\mathbf{H}^{\frac{1}{2}}}+\|\nabla \times A \mathbf{E}\|_{\mathbf{H}^{\frac{1}{2}}} \leq c\|\mathbf{E}\|_{\mathbf{L}^{2}} .
$$

Proof. The $\mathbf{H}^{\frac{1}{2}}$-estimate on $A \mathbf{E}$ is a consequence of Lemma 2.1. Let us now show that a similar estimate holds for $\nabla \times A \mathbf{E}$. Using (2.4), i.e., $\nabla \times \nabla \times A \mathbf{E}+\nabla p=\mathbf{E}$ with $p \in H_{0}^{1}(\Omega)$, we infer that $\nabla \times(\nabla \times A \mathbf{E}) \in \mathbf{L}^{2}(\Omega)$. Moreover, the boundary condition $A \mathbf{E} \times\left.\mathbf{n}\right|_{\partial \Omega}=0$ implies $\left.(\nabla \times A \mathbf{E}) \cdot \mathbf{n}\right|_{\partial \Omega}=0$. In conclusion $\nabla \times A \mathbf{E}$ is a member of $\mathbf{H}_{0}(\operatorname{div}, \Omega) \cap \mathbf{H}(\operatorname{curl}, \Omega)$ with $\|\nabla \times A \mathbf{E}\|_{\mathbf{L}^{2}}+\|\nabla \times \nabla \times A \mathbf{E}\|_{\mathbf{L}^{2}} \leq c\|\mathbf{E}\|_{\mathbf{L}^{2}}$, which, owing to Remark 2.1 implies the result.

We now construct an extension operator over $\mathbb{R}^{d}$ in order to regularize $A \mathbf{E}$ by mollification. This is the subject of the next lemma. For any real number $a$, the notation $a^{-}$henceforth stands for any real number strictly smaller than $a$.

Lemma 2.3 (Extension). Let $0 \leq s \leq \frac{1}{2}^{-}$. There is $c>0$ so that for any $\mathbf{F} \in \mathbf{H}_{0}(\operatorname{curl}, \Omega) \cap \mathbf{H}^{s}(\Omega)$ with $\nabla \times \mathbf{F} \in \mathbf{H}^{s}(\Omega)$, there exist an extension $\widetilde{\mathbf{F}}: \mathbb{R}^{d} \rightarrow \mathbb{R}^{d}$ satisfying

$$
\begin{aligned}
& \|\widetilde{\mathbf{F}}\|_{\mathbf{H}^{s}\left(\mathbb{R}^{d}\right)} \leq c\|\mathbf{F}\|_{\mathbf{H}^{s}(\Omega)}, \\
& \|\nabla \times \widetilde{\mathbf{F}}\|_{\mathbf{H}^{s}\left(\mathbb{R}^{d}\right)} \leq c\|\nabla \times \mathbf{F}\|_{\mathbf{H}^{s}(\Omega)} .
\end{aligned}
$$

Proof. Let $\widetilde{\mathbf{F}}$ be the extension by 0 of $\mathbf{F}$. The estimate (2.8) is a direct consequence of the property $\mathbf{H}_{0}^{s}(\Omega)=\mathbf{H}^{s}(\Omega)$ for $s<\frac{1}{2}$; see Section 2.1. The estimate (2.9) is obtained similarly once one realizes that

$$
\nabla \times \widetilde{\mathbf{F}}=\widetilde{\nabla \times \mathbf{F}} \in \mathbf{H}^{s}\left(\mathbb{R}^{d}\right) .
$$


Using $\mathbf{L}^{2}\left(\mathbb{R}^{d}\right)$ as pivot space, the above equality is proved by observing that

$$
\begin{aligned}
\langle\nabla \times \widetilde{\mathbf{F}}, \boldsymbol{\psi}\rangle_{s,-s} & =\int_{\mathbb{R}^{d}} \widetilde{\mathbf{F}} \cdot \nabla \times \boldsymbol{\psi}=\int_{\Omega} \mathbf{F} \cdot \nabla \times \boldsymbol{\psi}=\int_{\Omega} \nabla \times \mathbf{F} \cdot \boldsymbol{\psi}=\int_{\mathbb{R}^{d}} \widetilde{\nabla \times \mathbf{F}} \cdot \boldsymbol{\psi} \\
& =\langle\widehat{\nabla \times \mathbf{F}}, \boldsymbol{\psi}\rangle_{s,-s}
\end{aligned}
$$

holds for all $\boldsymbol{\psi} \in C_{0}^{\infty}\left(\mathbb{R}^{d}\right)$. This completes the proof.

The regularity estimate (2.7) together with the stability of the extension provided by Lemma 2.3 are key ingredients for our analysis. For approximation purposes we will need to mollify $A \mathbf{E}$ and we must make sure that the estimate (2.7) is stable by mollification. Let $\epsilon>0$ be a small parameter, yet to be chosen (see Lemmas 3.3. 4.2, and (5.4), and let us set

$$
\rho_{\epsilon}(\mathbf{x})=\epsilon^{-d} \rho(\epsilon \mathbf{x}), \quad \text { where } \quad \rho(\mathbf{x}):= \begin{cases}\eta \exp \left(-1 /\left(1-|\mathbf{x}|^{2}\right)\right), & \text { if }|\mathbf{x}|<1, \\ 0, & |\mathbf{x}| \geq 1,\end{cases}
$$

where $\eta$ is defined such that $\int_{\mathbb{R}^{d}} \rho(\mathbf{x})=1$.

We now define for all $\mathbf{E} \in \mathbf{L}^{2}(\Omega)$ the regularization of $A \mathbf{E}$ by

$$
(A \mathbf{E})_{\epsilon}:=\left.\left(\rho_{\epsilon} * \widetilde{A \mathbf{E}}\right)\right|_{\Omega},
$$

where $\widetilde{A \mathbf{E}}$ denotes the extension of $A \mathbf{E}$ over $\mathbb{R}^{d}$ provided by Lemma 2.3 and $\left.\cdot\right|_{\Omega}$ denotes the restriction to $\Omega$.

The following lemma gathers the main approximation results that we shall need in the rest of the paper.

Lemma 2.4 (Approximation by Smooth Functions). There is a constant $c$, only depending on $\Omega$, so that for all $\mathbf{E} \in \mathbf{L}^{2}(\Omega)$,

$$
\begin{array}{rlrl}
\left\|A \mathbf{E}-(A \mathbf{E})_{\epsilon}\right\|_{\mathbf{H}^{s}} & \leq c \epsilon^{\frac{1}{2}^{-}-s}\|A \mathbf{E}\|_{\mathbf{H}^{\frac{1}{2}}}, & & 0 \leq s \leq \frac{1}{2}^{-}, \\
\left\|\nabla \times\left(A \mathbf{E}-(A \mathbf{E})_{\epsilon}\right)\right\|_{\mathbf{L}^{2}} & \leq c \epsilon^{\frac{1}{2}^{-}}\|\nabla \times A \mathbf{E}\|_{\mathbf{H}^{\frac{1}{2}}}, & & \\
\left\|(A \mathbf{E})_{\epsilon}\right\|_{\mathbf{H}^{s}} & \leq c \epsilon^{-s \frac{1}{2}^{-}}\|A \mathbf{E}\|_{\mathbf{H}^{\frac{1}{2}}}, & \frac{1}{2}^{-} \leq s .
\end{array}
$$

Proof. Let us first observe that the Riesz-Thorin interpolation theorem implies that $\left\|\left.f\right|_{\Omega}\right\|_{H^{s}(\Omega)} \leq\|f\|_{H^{s}\left(\mathbb{R}^{d}\right)}$ for all $f \in H^{s}\left(\mathbb{R}^{d}\right)$. Using the estimate (2.8) we then proceed as follows to prove (2.11):

$$
\begin{aligned}
\left\|A \mathbf{E}-(A \mathbf{E})_{\epsilon}\right\|_{\mathbf{H}^{s}(\Omega)} & =\left\|\left.\widetilde{A \mathbf{E}}\right|_{\Omega}-(A \mathbf{E})_{\epsilon}\right\|_{\mathbf{H}^{s}(\Omega)} \leq\left\|\widetilde{A \mathbf{E}}-\rho_{\epsilon} * \widetilde{A \mathbf{E}}\right\|_{\mathbf{H}^{s}\left(\mathbb{R}^{d}\right)} \\
& \leq c \epsilon^{\frac{1^{2}}{}{ }^{-}-s}\|\widetilde{A \mathbf{E}}\|_{\mathbf{H}^{\frac{1}{2}}{ }^{-}\left(\mathbb{R}^{d}\right)} \leq c^{\epsilon^{\frac{1^{2}}{}-s}\|A \mathbf{E}\|_{\mathbf{H}^{\frac{1}{2}}{ }^{-}}(\Omega)} .
\end{aligned}
$$

We refer, for instance, to [26, Chapter 7] and [25. Appendix C] for more details on the approximation properties of the mollification operator. Using (2.10) and the property of the convolution product $\nabla \times\left(\rho_{\epsilon} * \widetilde{A \mathbf{E}}\right)=\rho_{\epsilon} * \nabla \times \widetilde{A \mathbf{E}}$, we prove (2.12) as follows:

$$
\begin{aligned}
\left\|\nabla \times\left(A \mathbf{E}-(A \mathbf{E})_{\epsilon}\right)\right\|_{\mathbf{L}^{2}(\Omega)} & \left.=\| \widetilde{(\nabla \times A \mathbf{E}}-\nabla \times \rho_{\epsilon} * \widetilde{A \mathbf{E}}\right)\left.\right|_{\Omega} \|_{\mathbf{L}^{2}(\Omega)} \\
& \leq\left\|\widetilde{\nabla \times A \mathbf{E}}-\nabla \times \rho_{\epsilon} * \widetilde{A \mathbf{E}}\right\|_{\mathbf{L}^{2}\left(\mathbb{R}^{d}\right)} \\
& =\left\|\nabla \times \widetilde{A \mathbf{E}}-\rho_{\epsilon} * \nabla \times \widetilde{A \mathbf{E}}\right\|_{\mathbf{L}^{2}\left(\mathbb{R}^{d}\right)} \leq c \epsilon^{\frac{1}{2}}\|A \mathbf{E}\|_{\mathbf{H}^{\frac{1}{2}}{ }^{-}(\Omega)} .
\end{aligned}
$$


Finally, we derive (2.13) by observing again that

$$
\left\|(A \mathbf{E})_{\epsilon}\right\|_{\mathbf{H}^{s}(\Omega)}=\left\|\left.\rho_{\epsilon} * \widetilde{A \mathbf{E}}\right|_{\Omega}\right\|_{\mathbf{H}^{s}\left(\mathbb{R}^{d}\right)} \leq\left\|\rho_{\epsilon} * \widetilde{A \mathbf{E}}\right\|_{\mathbf{H}^{s}\left(\mathbb{R}^{d}\right)},
$$

and we conclude by using standard inverse estimates (see [25, Appendix C]) and estimate (2.8).

2.4. Continuous Lagrange elements. Let $\left\{\mathcal{T}_{h}\right\}_{h>0}$ be a shape regular sequence of subdivisions of $\Omega$. Associated with the mesh family $\left\{\mathcal{T}_{h}\right\}_{h>0}$ we assume that we have at hand two families of finite-dimensional vector spaces $\left\{\mathbf{X}_{h}\right\}_{h>0}$ and $\left\{M_{h}\right\}_{h>0}$ conforming in $\mathbf{H}^{1}(\Omega) \cap \mathbf{H}_{0}(\operatorname{curl}, \Omega)$ and $H_{0}^{1}(\Omega)$, respectively.

The space $\mathbf{X}_{h}$ will be used to approximate the vector field $\mathbf{E}$, whereas $M_{h}$ will be used to approximate the Lagrange multiplier associated with the divergence-free constraint.

We assume that the sequence $\left\{\mathbf{X}_{h}\right\}_{h>0}$ is such that there exists a family of operators $\mathcal{C}_{h}: \mathbf{H}_{0}(\operatorname{curl}, \Omega) \longrightarrow \mathbf{X}_{h} \subset \mathbf{H}_{0}(\operatorname{curl}, \Omega)$, satisfying the following stability and approximation properties: There exist $r \geq 2$ and $c$, uniform in $h$, so that for every $\mathbf{F}$ in $\mathbf{H}^{l}(\Omega) \cap \mathbf{H}_{0}(\operatorname{curl}, \Omega)$

$$
\begin{aligned}
\left\|\mathcal{C}_{h} \mathbf{F}\right\|_{\mathbf{H}^{l}} & \leq c\|\mathbf{F}\|_{\mathbf{H}^{l}}, \quad 0 \leq l<\frac{3}{2}, \\
\left\|\mathbf{F}-\mathcal{C}_{h} \mathbf{F}\right\|_{\mathbf{H}^{t}}, & \leq c h^{l-t}\|\mathbf{F}\|_{\mathbf{H}^{l}} \quad 0 \leq t \leq l \leq r, \quad t<\frac{3}{2} .
\end{aligned}
$$

The operator $\mathcal{C}_{h}$ can be the Clément [16] or the Scott-Zhang 40 interpolation operator when the discrete space $\mathbf{X}_{h}$ is constructed using finite elements. Abusing the notation, we also assume that there is a family of operators $\mathcal{C}_{h}: H_{0}^{1}(\Omega) \longrightarrow$ $M_{h} \subset H_{0}^{1}(\Omega)$ satisfying the scalar-valued counterparts of (2.14)-(2.15).

Remark 2.3 (Approximation by Finite Elements). The limit $l<\frac{3}{2}$ in (2.14) corresponds to the best that can be achieved with $\mathcal{C}^{0}$-Lagrange finite elements. The parameter $r$ in (2.15) corresponds to the limit imposed by using Lagrange elements of polynomial degree at most $r-1$.

\section{The $H^{-\alpha}$ PENALTY}

We propose and analyze in this section an approximation method based on the control of the $H^{-\alpha}$-norm of the divergence.

3.1. Motivation. When looking closely at (1.2), one notices that the eigenfunctions associated with non-zero eigenvalues are necessarily divergence free, since by applying the divergence operator to both sides of (1.2) one obtains $0=\lambda \nabla \cdot \mathbf{E}$. As a result the constraint $\nabla \cdot \mathbf{E}=0$ is redundant at the continuous level for non-zero eigenvalues, and it makes sense to consider the following alternative eigenvalue problem: Find $\mathbf{E} \in \mathbf{H}_{0}(\operatorname{curl}, \Omega) \backslash\{0\}$ and $\lambda \neq 0$ so that

$$
\nabla \times \nabla \times \mathbf{E}=\lambda \mathbf{E} .
$$

Most standard approximation techniques for (1.2) are based on the formulation (3.1) or a mixed form thereof which introduces a Lagrange multiplier to enforce the divergence-free constraint. All of these methods are more or less equivalent and rely on two key hypotheses: (i) there exists a family of discrete $\left\{M_{h}\right\}_{h>0}$ so that $\nabla M_{h} \subset \mathbf{X}_{h}$; (ii) the discrete compactness property holds (A sequence $\left\{\mathbf{E}_{h}\right\}$ of functions in $\mathbf{X}_{h}$ satisfying $\left(\mathbf{E}_{h}, \nabla q_{h}\right)=0$ for all $q_{h} \in M_{h}$ is said to satisfy the discrete compactness property if there exists a subsequence converging strongly to 
a limit in $\mathbf{L}^{2}(\Omega)$.) The two hypotheses (i) and (ii) are tailored to recover some compactness from the discrete versions of (3.1). We refer to [20, 29] for review papers; see also [3, 11, 13, 21, 37, 31, 8, 9]. The bottom line is that only $\mathbf{H}$ (curl)-conforming edge finite elements are known to satisfy (i) and (ii). Some Discontinuous Galerkin approximations on conforming meshes are also appropriate provided the discontinuous approximation space contains a $\mathbf{H}$ (curl)-conforming edge finite elements subspace [12].

Note that the hypothesis (i) excludes the use of Lagrange finite elements to construct the approximation space $\mathbf{X}_{h}$ unless the space of Lagrange multipliers $M_{h}$ is composed of elements of class $\mathcal{C}^{1}$. Despite this obstacle, we nevertheless pursue our idea of using $\mathcal{C}^{0}$-Lagrange finite elements. Actually, a significant contribution in this direction has been made by Dauge and Costabel in [19. The main idea defended in [19] consists of abandoning (3.1) and to re-introduce the divergencefree constraint by penalizing it in an appropriate norm. More precisely, the authors propose to replace (3.1) by the following problem: Find $\mathbf{E} \in \mathbf{H}_{0}(\operatorname{curl}, \Omega) \backslash\{0\}$ and $\lambda$ so that

$$
\nabla \times \nabla \times \mathbf{E}-\nabla(P(\nabla \cdot \mathbf{E}))=\lambda \mathbf{E},
$$

where the operator $P$ is appropriately defined. Using the identity for $P$ and weakly enforcing the boundary condition $\left.P(\nabla \cdot \mathbf{E})\right|_{\partial \Omega}=0$ is an easy fix (at least for the boundary value problem (1.1) with $\omega=0$ ), but it is also a bad idea when $\mathbf{X}_{h}$ is composed of $\mathcal{C}^{0}$-Lagrange finite elements, since it implies that any solution to (3.2) satisfies a uniform bound in $\mathbf{H}_{0}(\operatorname{curl}, \Omega) \cap \mathbf{H}(\operatorname{div}, \Omega)$; see e.g. [28, 34]. It is known since the ground-breaking work of Costabel [18, that any $\mathbf{H}^{1}$-conforming method that is uniformly stable in $\mathbf{H}_{0}(\operatorname{curl}, \Omega) \cap \mathbf{H}(\operatorname{div}, \Omega)$ cannot converge if $\Omega$ is nonsmooth and non-convex. The main reason for the failure is that $\mathbf{H}^{1}(\Omega) \cap \mathbf{H}_{0}(\operatorname{curl}, \Omega)$ is a closed proper subspace of $\mathbf{H}_{0}(\operatorname{curl}, \Omega) \cap \mathbf{H}(\operatorname{div}, \Omega)$ when $\Omega$ has re-entrant corners. The key of the method proposed in [19] is to construct an operator $P$ that controls $\nabla \cdot \mathbf{E}$ in a weighted Sobolev space that is intermediate between $L^{2}(\Omega)$ and $H^{-1}(\Omega)$. More precisely, $P$ is a projection on a weighted $L^{2}$-space where the weight is a distance to the re-entrant corners to some appropriate power. The mixed version of this idea has been shown to be spectrally correct in [10. We refer to [14, 15] for further elaboration on this idea and related implementation issues.

To summarize the situation, controlling the divergence in $L^{2}(\Omega)$ is too strong to ensure pointwise approximation, while controlling it in $H^{-1}(\Omega)$ or in a weighted $L^{2}$-space is too weak to guarantee collective compactness. The idea that we propose to explore in the present paper consists of penalizing the divergence in the space $H^{-\alpha}(\Omega)$ with $\alpha \in\left(\frac{1}{2}, 1\right)$. We then introduce the following Hilbert space

$$
\mathbf{X}^{-\alpha}=\left\{\mathbf{v} \in \mathbf{H}_{0}(\operatorname{curl}, \Omega) ; \nabla \cdot \mathbf{v} \in \mathbf{H}^{-\alpha}(\Omega)\right\} .
$$

Lemma 3.1 $\left(\mathbf{H}^{1-\alpha}(\Omega)\right.$ Estimate). Let $\Omega$ be a bounded, simply connected, Lipschitz domain in $\mathbb{R}^{d}, d=2,3$. For any $\alpha \in\left(\frac{1}{2}, 1\right]$, there is $c>0$ so that the following holds for all $\mathbf{F} \in \mathbf{X}^{-\alpha}$ :

$$
c\|\mathbf{F}\|_{\mathbf{H}^{1-\alpha}} \leq\|\nabla \times \mathbf{F}\|_{\mathbf{L}^{2}}+\|\nabla \cdot \mathbf{F}\|_{H^{-\alpha}} .
$$

Proof. Consider $\mathbf{F} \in \mathbf{X}^{-\alpha}$ and define $p \in H_{0}^{1}(\Omega)$ solving

$$
(\nabla p, \nabla q)=(\mathbf{F}, \nabla q), \quad \forall q \in H_{0}^{1}(\Omega) .
$$


Since $\alpha \in\left(\frac{1}{2}, \frac{3}{2}\right)$, the regularity estimate from Theorem 2.1 implies the existence of a constant $c>0$ so that $\|p\|_{H^{2-\alpha}(\Omega)} \leq c\|\nabla \cdot \mathbf{F}\|_{H^{-\alpha}(\Omega)}$. Let us set $\mathbf{v}=\mathbf{F}-\nabla p$. The function $\mathbf{v}$ satisfies $\nabla \cdot \mathbf{v}=0, \mathbf{v} \times\left.\mathbf{n}\right|_{\partial \Omega}=0$ since $\nabla \cdot \mathbf{F}=\Delta p$, and $\mathbf{F} \times\left.\mathbf{n}\right|_{\partial \Omega}=0=$ $\nabla p \times\left.\mathbf{n}\right|_{\partial \Omega}$. Then using the fact that $\Omega$ is simply connected together with (2.2) we deduce that there exists a constant $c$ so that

$$
c\|\mathbf{v}\|_{\mathbf{H}^{\frac{1}{2}}} \leq\|\nabla \times \mathbf{v}\|_{\mathbf{L}^{2}}=\|\nabla \times \mathbf{F}\|_{\mathbf{L}^{2}} .
$$

In conclusion, we obtain

$$
\|\mathbf{F}\|_{\mathbf{H}^{1-\alpha}(\Omega)} \leq\|\nabla p\|_{\mathbf{H}^{1-\alpha}}+\|\mathbf{v}\|_{\mathbf{H}^{\frac{1}{2}}} \leq c\left(\|\nabla \cdot \mathbf{F}\|_{H^{-\alpha}}+\|\nabla \times \mathbf{F}\|_{\mathbf{L}^{2}}\right),
$$

and this completes the proof.

An immediate consequence of (3.4) is that $\mathbf{X}^{-\alpha}$ is continuously embedded in $\mathbf{H}^{1-\alpha}(\Omega)$. Upon denoting $\langle\cdot, \cdot\rangle_{-\alpha}$ the $H^{-\alpha}$-scalar product defined in (2.1), the rest of the paper hinges on the idea that the bilinear form

$$
a_{\alpha}(\mathbf{E}, \mathbf{F}):=(\nabla \times \mathbf{E}, \nabla \times \mathbf{F})+\langle\nabla \cdot \mathbf{E}, \nabla \cdot \mathbf{F}\rangle_{H^{-\alpha}}
$$

is coercive on $\mathbf{X}^{-\alpha}$.

Remark 3.1 (Incomplete Consistency). Let $A_{\alpha}: \mathbf{X}^{-\alpha} \longrightarrow\left(\mathbf{X}^{-\alpha}\right)^{\prime}$ be the operator defined by $\left\langle A_{\alpha} \mathbf{E}, \mathbf{F}\right\rangle:=a_{\alpha}(\mathbf{E}, \mathbf{F})$. It is clear that if $(\mathbf{E}, 1 / \lambda)$ is an eigenpair of $A$ (i.e., $(\mathbf{E}, \lambda)$ is an eigenpair of (1.2) $)$, then $(\mathbf{E}, 1 / \lambda)$ is an eigenpair of $A_{\alpha}$, but the converse is not true. Let $(\psi, \mu)$ be an eigenpair of $\left(-\Delta_{D}\right)^{1-\alpha}$ and let $\mathbf{E}:=\nabla\left(-\Delta_{D}\right)^{-1} \psi$, then

$$
-\nabla\left(-\Delta_{D}\right)^{-\alpha} \nabla \cdot \mathbf{E}=\nabla\left(-\Delta_{D}\right)^{-\alpha} \psi=\nabla\left(-\Delta_{D}\right)^{-1}\left(-\Delta_{D}\right)^{1-\alpha} \psi=\mu \mathbf{E},
$$

thereby proving that $(\mathbf{E}, 1 / \mu)$ is an eigenpair of $A_{\alpha}$. Since $\nabla \cdot \mathbf{E}=-\psi \neq 0$, this construction proves that $(\mathbf{E}, 1 / \mu)$ is not an eigenpair of $A$. This also shows that the bilinear form $a_{\alpha}$ is not appropriate to approximate the spectrum of $A$; nevertheless, we proceed and will correct this inconsistency in $\$ 5$.

3.2. Formulation of the $\mathbf{H}^{-\alpha}$ penalty. We consider in this section the following discrete formulation of (1.2): Seek $\mathbf{E}_{h} \in \mathbf{X}_{h} \backslash\{0\}$ and $\lambda_{h} \in \mathbb{R}$ so that for all $\mathbf{F}_{h} \in \mathbf{X}_{h}$,

$$
a_{\alpha}\left(\mathbf{E}_{h}, \mathbf{F}_{h}\right)=\lambda_{h}\left(\mathbf{E}_{h}, \mathbf{F}_{h}\right) .
$$

The above problem is not easy to implement due to the presence of the non-trivial scalar product $\langle\cdot, \cdot\rangle_{-\alpha}$; nevertheless, we concentrate our attention on this problem since it is the basis for two relaxed formulations proposed in the following sections.

Let us introduce the following norm:

$$
\|\mathbf{F}\|:=\|\mathbf{F}\|_{\mathbf{H}^{1-\alpha}}+\|\nabla \times \mathbf{F}\|_{\mathbf{L}^{2}}+\|\nabla \cdot \mathbf{F}\|_{H^{-\alpha}} .
$$

The basic stability and boundedness properties of the bilinear form $a_{\alpha}$ with respect to this norm are gathered in the following lemma.

Lemma 3.2 (Coercivity and Continuity of $\left.a_{\alpha}\right)$. Let $\alpha \in\left(\frac{1}{2}, 1\right)$. The bilinear form $a_{\alpha}: \mathbf{X}^{-\alpha} \times \mathbf{X}^{-\alpha} \rightarrow \mathbb{R}$ satisfies

$$
c\|\mathbf{F}\|^{2} \leq a_{\alpha}(\mathbf{F}, \mathbf{F}) \quad \text { and } \quad a_{\alpha}(\mathbf{F}, \mathbf{E}) \leq\|\mathbf{E}\|\|\mathbf{F}\|, \quad \forall \mathbf{E}, \mathbf{F} \in \mathbf{X}^{-\alpha},
$$

where the constant c solely depends on $\Omega$.

Proof. The left estimate in (3.8) is a direct consequence of Lemma 3.1. The right estimate in (3.8) readily follows from the Cauchy-Schwarz inequality. 
We now define the discrete operator associated with $A_{\alpha}$, say $A_{h}: \mathbf{L}^{2}(\Omega) \rightarrow \mathbf{X}_{h}$ : $\mathbf{E} \mapsto A_{h} \mathbf{E}$, by defining $A_{h} \mathbf{E}$ to be the unique element in $\mathbf{X}_{h}$ satisfying

$$
a_{\alpha}\left(A_{h} \mathbf{E}, \mathbf{F}_{h}\right)=\left(\mathbf{E}, \mathbf{F}_{h}\right), \quad \forall \mathbf{F}_{h} \in \mathbf{X}_{h} .
$$

It is clear that $A_{h}$ is self-adjoint. The discrete eigenvalue problem (3.6) can then be recast as follows: Seek $\mathbf{E}_{h} \in \mathbf{X}_{h}$ and $\lambda_{h} \in \mathbb{R}$ so that

$$
A_{h} \mathbf{E}_{h}=\frac{1}{\lambda_{h}} \mathbf{E}_{h} \text {. }
$$

3.3. Incomplete pointwise convergence and collective compactness of the $\mathbf{H}^{-\alpha}$ penalty. The convergence analysis of the discrete problem (3.6) is done by proving an incomplete pointwise convergence property and the collective compactness.

Lemma 3.3 (Incomplete Pointwise Convergence). Let $\alpha \in\left(\frac{1}{2}, 1\right]$. The sequence $\left\{A_{h}\right\}_{h>0}$ converges pointwise to $A$ in $\mathbf{H}(\operatorname{div}=0, \Omega)$. More precisely, there exists a constant $c$ independent of the mesh size $h$ such that for any $\mathbf{E} \in \mathbf{H}(\operatorname{div}=0, \Omega)$ the following estimate holds:

$$
\left\|A \mathbf{E}-A_{h} \mathbf{E}\right\|_{\mathbf{L}^{2}} \leq \operatorname{ch}^{\left(\left(\alpha-\frac{1}{2}\right) \frac{r-1}{\alpha+r-1}\right)^{-}}\|\mathbf{E}\|_{\mathbf{L}^{2}}, \quad \forall 0<h<1 .
$$

Proof. Let $\mathbf{E}$ be a vector field in $\mathbf{L}^{2}(\Omega)$; we want to prove that $A_{h} \mathbf{E}$ converges to $A \mathbf{E}$ in $\mathbf{L}^{2}(\Omega)$ as $h$ goes to zero. We start by dividing the difference $A_{h} \mathbf{E}-A \mathbf{E}$ into three terms,

$$
\begin{gathered}
\left\|A \mathbf{E}-A_{h} \mathbf{E}\right\|_{\mathbf{L}^{2}} \leq\left\|A \mathbf{E}-(A \mathbf{E})_{\epsilon}\right\|_{\mathbf{L}^{2}}+\left\|(A \mathbf{E})_{\epsilon}-\mathcal{C}_{h}(A \mathbf{E})_{\epsilon}\right\|_{\mathbf{L}^{2}} \\
+\left\|\mathcal{C}_{h}(A \mathbf{E})_{\epsilon}-A_{h} \mathbf{E}\right\|_{\mathbf{L}^{2}}
\end{gathered}
$$

where $(A \mathbf{E})_{\epsilon}$ is the mollified approximation of $A \mathbf{E}$ defined in Section 2.3 and $\mathcal{C}_{h}$ is the approximation operator defined in Section 2.4. We now bound individually the three terms in the right-hand side of (3.12).

For the first term, we directly obtain from (2.11) with $s=0$ that

$$
\left\|A \mathbf{E}-(A \mathbf{E})_{\epsilon}\right\|_{\mathbf{L}^{2}} \leq c \epsilon^{\frac{1}{2}}\|A \mathbf{E}\|_{\mathbf{H}^{\frac{1}{2}}} .
$$

For the second term, we use the approximation estimate (2.15) with $l=\frac{1}{2}^{-}$and $t=0$ and the stability estimate (2.13) with $s=\frac{1}{2}^{-}$to obtain

$$
\left\|(A \mathbf{E})_{\epsilon}-\mathcal{C}_{h}(A \mathbf{E})_{\epsilon}\right\|_{\mathbf{L}^{2}} \leq c h^{\frac{1}{2}^{-}}\|A \mathbf{E}\|_{\mathbf{H}^{\frac{1}{2}}} .
$$

Bounding the third term is more technical. Recalling the definition of the norm $\|\cdot\|$ (see (3.7)) it suffices to bound $\left\|\mathcal{C}_{h}(A \mathbf{E})_{\epsilon}-A_{h} \mathbf{E}\right\|$. Using the coercivity and the continuity (3.8) together with the Galerkin orthogonality (valid since $\nabla \cdot \mathbf{E}=0$ )

$$
a_{\alpha}\left(A \mathbf{E}-A_{h} \mathbf{E}, \mathcal{C}_{h}(A \mathbf{E})_{\epsilon}-A_{h} \mathbf{E}\right)=0,
$$

we deduce that

$$
\begin{aligned}
c\left\|\mathcal{C}_{h}(A \mathbf{E})_{\epsilon}-A_{h} \mathbf{E}\right\|^{2} & \leq a_{\alpha}\left(\mathcal{C}_{h}(A \mathbf{E})_{\epsilon}-A_{h} \mathbf{E}, \mathcal{C}_{h}(A \mathbf{E})_{\epsilon}-A_{h} \mathbf{E}\right) \\
& \leq a_{\alpha}\left(\mathcal{C}_{h}(A \mathbf{E})_{\epsilon}-A \mathbf{E}, \mathcal{C}_{h}(A \mathbf{E})_{\epsilon}-A_{h} \mathbf{E}\right) \\
& \leq\left\|\mathcal{C}_{h}(A \mathbf{E})_{\epsilon}-A \mathbf{E}\right\|\left\|\mathcal{C}_{h}(A \mathbf{E})_{\epsilon}-A_{h} \mathbf{E}\right\| .
\end{aligned}
$$

As a result,

$$
c\left\|\mathcal{C}_{h}(A \mathbf{E})_{\epsilon}-A_{h} \mathbf{E}\right\| \leq\left\|\mathcal{C}_{h}(A \mathbf{E})_{\epsilon}-(A \mathbf{E})_{\epsilon}\right\|+\left\|(A \mathbf{E})_{\epsilon}-A \mathbf{E}\right\| .
$$


Lemma 3.1 and the approximation estimate 2.15) with $t=1, l=r$ together with the inverse estimate (2.13) with $s=r$, yield

$$
\left\|\mathcal{C}_{h}(A \mathbf{E})_{\epsilon}-(A \mathbf{E})_{\epsilon}\right\| \leq c\left\|\mathcal{C}_{h}(A \mathbf{E})_{\epsilon}-(A \mathbf{E})_{\epsilon}\right\|_{\mathbf{H}^{1}} \leq c^{\prime} h^{r-1} \epsilon^{\left(-r+\frac{1}{2}\right)^{-}}\|A \mathbf{E}\|_{\mathbf{H}^{\frac{1}{2}}} .
$$

The error estimate (2.12) together with the error estimate (2.11) with $s=1-\alpha$ implies

$$
\begin{aligned}
\left\|(A \mathbf{E})_{\epsilon}-A \mathbf{E}\right\| & \leq\left\|\nabla \times\left((A \mathbf{E})_{\epsilon}-A \mathbf{E}\right)\right\|_{\mathbf{L}^{2}}+c\left\|(A \mathbf{E})_{\epsilon}-A \mathbf{E}\right\|_{\mathbf{H}^{1-\alpha}} \\
& \leq c_{1} \epsilon^{\frac{1}{2}^{-}}\|\nabla \times A \mathbf{E}\|_{\mathbf{H}^{\frac{1}{2}^{-}}}+c_{2} \epsilon^{\left(\alpha-\frac{1}{2}\right)^{-}}\|A \mathbf{E}\|_{\mathbf{H}^{1^{2}}} .
\end{aligned}
$$

Gathering the above estimates, invoking the stability estimate (2.7), and choosing $\epsilon=h^{\frac{r-1}{\alpha+r-1}}$ for $h \leq 1$, we arrive at (3.11). The result follows by taking the limit $h \rightarrow 0$ recalling that $\alpha \in\left(\frac{1}{2}, 1\right]$.

Lemma 3.4 (Collective Compactness). Let $\alpha \in\left(\frac{1}{2}, 1\right)$. The sequence $\mathcal{A}:=\left\{A_{h}\right\}_{h>0}$ is collectively compact.

Proof. Let $U$ be a bounded set of $\mathbf{L}^{2}(\Omega)$. We must prove that the image set

$$
\mathcal{A} U=\left\{A_{h} \mathbf{E} ; \mathbf{E} \in U, A_{h} \in \mathcal{A}\right\}
$$

is relatively compact in $\mathbf{L}^{2}(\Omega)$. Let $\mathbf{E}$ be a member of $U$. The coercivity of the bilinear form $a_{\alpha}$ and the definition of the operator $A_{h}$ imply that

$$
c\left\|A_{h} \mathbf{E}\right\|_{\mathbf{H}^{1-\alpha}}^{2} \leq a_{\alpha}\left(A_{h} \mathbf{E}, A_{h} \mathbf{E}\right)=\left(\mathbf{E}, A_{h} \mathbf{E}\right) \leq\left\|A_{h} \mathbf{E}\right\|_{\mathbf{H}^{1-\alpha}}\|\mathbf{E}\|_{\mathbf{H}^{\alpha-1}} .
$$

Note that the last inequality is a consequence of the fact that $\mathbf{H}^{\alpha-1}(\Omega)=\left[\mathbf{H}_{0}^{1-\alpha}(\Omega)\right]^{\prime}$ and $\mathbf{H}_{0}^{1-\alpha}(\Omega)=\mathbf{H}^{1-\alpha}(\Omega)$ since $1-\alpha \in\left(0, \frac{1}{2}\right)$ (see 2.1 for the definition of $\mathbf{H}_{0}^{s}(\Omega)$ ). We then deduce that

$$
c\left\|A_{h} \mathbf{E}\right\|_{\mathbf{H}^{1-\alpha}} \leq\|\mathbf{E}\|_{\mathbf{H}^{\alpha-1}} .
$$

Let $\left\{\mathbf{E}_{n}\right\}$ be a sequence in $U \subset \mathbf{L}^{2}(\Omega)$. One can extract a subsequence $\left\{\mathbf{E}_{n_{k}}\right\}$ that converges weakly in $\mathbf{L}^{2}(\Omega)$. This subsequence converges strongly in $\mathbf{H}^{\alpha-1}(\Omega)$ since the embedding $\mathbf{L}^{2}(\Omega) \subset \mathbf{H}^{\alpha-1}(\Omega)$ is compact. The inequality (3.13) implies that the subsequence $\left\{A_{h} \mathbf{E}_{n_{k}}\right\}$ converges strongly in $\mathbf{H}^{1-\alpha}(\Omega)$, which also implies strong convergence in $\mathbf{L}^{2}(\Omega)$ since the embedding $\mathbf{H}^{1-\alpha}(\Omega) \subset \mathbf{L}^{2}(\Omega)$ is continuous.

Remark 3.2 (Convergence for Solenoidal Fields). The method is convergent for the boundary value problem (1.1) with $\omega=0$ since the right-hand side in (1.1) is necessarily divergence free. However, we cannot conclude that the algorithm is spectrally correct for the eigenvalue problem (1.2) since the pointwise convergence (see Lemma 3.3) is proved only for solenoidal fields, i.e., $\mathbf{E} \in \mathbf{H}(\operatorname{div}=0, \Omega)$. The

origin of this difficulty is that the method is consistent only if $\nabla \cdot \mathbf{E}=0$ (see also Remark 3.1). This issue is overcome in Section 5 by considering a mixed method.

\section{Relaxed $H^{-\alpha}$ PEnAlty}

We propose in this section to relax the penalty of the divergence in $H^{-\alpha}(\Omega)$ by using the $H^{-1}$-norm instead. The main justification for this change of point of view is that computing the $H^{-\alpha}$-norm is technical and requires a multi-scale decomposition of the approximation space $\mathbf{X}_{h}$ (see e.g. [7]), whereas approximating the $H^{-1}$-norm just requires solving scalar Poisson problems. 
4.1. Formulation of the relaxed $H^{-\alpha}$ penalty. Applying an inverse inequality gives

$$
\left\|\nabla \cdot \mathbf{F}_{h}\right\|_{H^{-\alpha}} \leq c h^{(\alpha-1)}\left\|\nabla \cdot \mathbf{F}_{h}\right\|_{H^{-1}}, \quad \forall \mathbf{F}_{h} \in \mathbf{X}_{h},
$$

which leads us to introduce the following bilinear form:

$$
a_{h}\left(\mathbf{E}_{h}, \mathbf{F}_{h}\right):=\left(\nabla \times \mathbf{E}_{h}, \nabla \times \mathbf{F}_{h}\right)+h^{2(\alpha-1)}\left\langle\nabla \cdot \mathbf{E}_{h}, \nabla \cdot \mathbf{F}_{h}\right\rangle_{H^{-1}} .
$$

We then consider the following discrete eigenvalue problem: Seek $\mathbf{E}_{h} \in \mathbf{X}_{h} \backslash\{0\}$ and $\lambda_{h} \in \mathbb{R}$ such that for all $\mathbf{F}_{h} \in \mathbf{X}_{h}$,

$$
a_{h}\left(\mathbf{E}_{h}, \mathbf{F}_{h}\right)=\lambda_{h}\left(\mathbf{E}_{h}, \mathbf{F}_{h}\right) .
$$

We shall see that the inverse estimate (4.1) is sufficient to prove an incomplete pointwise convergence result similar to (3.11). However, the bound in the other direction, namely $h^{(\alpha-1)}\left\|\nabla \cdot \mathbf{F}_{h}\right\|_{H^{-1}(\Omega)} \leq c\left\|\nabla \cdot \mathbf{F}_{h}\right\|_{H^{-\alpha}(\Omega)}$ does not hold in general. This observation will lead to the restriction $\alpha \in\left(\frac{r}{2 r-1}, 1\right)$ where $r \geq 2$ is defined in (2.15).

Let us introduce the following discrete norm

$$
\|\mathbf{F}\|_{h}:=\|\mathbf{F}\|_{\mathbf{H}^{1-\alpha}}+\|\nabla \times \mathbf{F}\|_{\mathbf{L}^{2}}+h^{\alpha-1}\|\nabla \cdot \mathbf{F}\|_{H^{-1}} .
$$

The basic stability and boundedness properties of the bilinear form $a_{h}(.,$.$) are$ summarized in the following:

Lemma 4.1 (Coercivity and Continuity of $\left.a_{h}\right)$. Let $\alpha \in\left(\frac{1}{2}, 1\right)$. There exists a uniform constant uniform $c$ so that

$$
\begin{aligned}
c\left\|\mathbf{F}_{h}\right\|_{h}^{2} & \leq a_{h}\left(\mathbf{F}_{h}, \mathbf{F}_{h}\right) & & \forall \mathbf{F}_{h} \in \mathbf{X}_{h}, \\
a_{h}(\mathbf{E}, \mathbf{F}) & \leq\|\mathbf{E}\|_{h}\|\mathbf{F}\|_{h} & & \forall \mathbf{E}, \mathbf{F} \in \mathbf{X}^{-\alpha} .
\end{aligned}
$$

Proof. The proof of (4.4) follows from Lemma 3.1 and (4.1). The inequality (4.5) is obtained by applying the Cauchy-Schwarz inequality.

We now abuse notation by reusing the symbol $A_{h}$ to define the discrete selfadjoint operator $A_{h}: \mathbf{L}^{2}(\Omega) \rightarrow \mathbf{X}_{h}: \mathbf{E} \mapsto A_{h} \mathbf{E}$, where $A_{h} \mathbf{E}$ is such that the following holds:

$$
a_{h}\left(A_{h} \mathbf{E}, \mathbf{F}_{h}\right)=\left(\mathbf{E}, \mathbf{F}_{h}\right), \quad \forall \mathbf{F}_{h} \in \mathbf{X}_{h} .
$$

Note that the existence and the uniqueness of $A_{h} \mathbf{E}$ are consequences of the coercivity and the boundedness of the bilinear form $a_{h}$ (see Lemma 4.1). Note also that $\left(\mathbf{E}_{h}, \lambda_{h}\right) \in \mathbf{X}_{h} \backslash\{0\} \times \mathbb{R} \backslash\{0\}$ is an eigenpair of (4.3) if and only if it satisfies

$$
A_{h} \mathbf{E}_{h}=\frac{1}{\lambda_{h}} \mathbf{E}_{h}
$$

4.2. Incomplete pointwise convergence and collective compactness of the relaxed $H^{-\alpha}$ penalty. The convergence analysis of the discrete eigenvalue problem (4.3) is done by proving the pointwise convergence property and the collective compactness.

Lemma 4.2 (Incomplete Pointwise Convergence). Let $r \geq 2$ be the restriction on the approximation estimate (2.15) and let $\alpha \in\left(\frac{r}{2 r-1}, 1\right]$. Then the sequence $\left\{A_{h}\right\}_{h>0}$ converges pointwise to $A$ in $\mathbf{H}(\operatorname{div}=0, \Omega)$. More precisely, there exists a constant $c$ independent of the mesh size $h$ such that for any $\mathbf{E} \in \mathbf{H}(\operatorname{div}=0, \Omega)$,

$$
\left\|A \mathbf{E}-A_{h} \mathbf{E}\right\|_{\mathbf{L}^{2}} \leq \operatorname{ch}^{\left(\alpha-\frac{1}{2}-\frac{\alpha}{2 r}\right)^{-}}\|\mathbf{E}\|_{\mathbf{L}^{2}}, \quad \forall h \in(0,1) .
$$


Proof. The proof is similar to that of Lemma 3.3 with $\|\cdot\|$ replaced by $\|\cdot\|_{h}$. Again, we have

$$
\begin{aligned}
\left\|A \mathbf{E}-A_{h} \mathbf{E}\right\|_{\mathbf{L}^{2}} & \leq\left\|A \mathbf{E}-(A \mathbf{E})_{\epsilon}\right\|_{\mathbf{L}^{2}}+\left\|(A \mathbf{E})_{\epsilon}-\mathcal{C}_{h}(A \mathbf{E})_{\epsilon}\right\|_{\mathbf{L}^{2}}+\left\|\mathcal{C}_{h}(A \mathbf{E})_{\epsilon}-A_{h} \mathbf{E}\right\|_{\mathbf{L}^{2}} \\
& \leq c\left({\epsilon^{\frac{1^{2}}{}}}^{-}+h^{1^{-}}\right)\|A \mathbf{E}\|_{\mathbf{H}^{1^{2}}}+\left\|\mathcal{C}_{h}(A \mathbf{E})_{\epsilon}-A_{h} \mathbf{E}\right\|_{\mathbf{L}^{2}},
\end{aligned}
$$

where $(A \mathbf{E})_{\epsilon}$ is the mollified approximation of $A \mathbf{E}$ defined in Section 2.3 and $\mathcal{C}_{h}$ is the approximation operator defined in Section 2.4. The coercivity and the boundedness of $a_{h}$ together with the Galerkin orthogonality imply

$$
\begin{aligned}
c\left\|\mathcal{C}_{h}(A \mathbf{E})_{\epsilon}-A_{h} \mathbf{E}\right\|_{\mathbf{L}^{2}} & \leq c\left\|\mathcal{C}_{h}(A \mathbf{E})_{\epsilon}-A_{h} \mathbf{E}\right\|_{h} \\
& \leq\left\|\mathcal{C}_{h}(A \mathbf{E})_{\epsilon}-(A \mathbf{E})_{\epsilon}\right\|_{h}+\left\|(A \mathbf{E})_{\epsilon}-A \mathbf{E}\right\|_{h} .
\end{aligned}
$$

The main difficulty consists of bounding from above the following two terms:

$$
\left\|(A \mathbf{E})_{\epsilon}-\mathcal{C}_{h}(A \mathbf{E})_{\epsilon}\right\|_{h} \quad \text { and } \quad\left\|A \mathbf{E}-(A \mathbf{E})_{\epsilon}\right\|_{h} .
$$

For the first term, we invoke the approximation estimates (2.15) together with (2.13) to claim

$$
\left\|(A \mathbf{E})_{\epsilon}-\mathcal{C}_{h}(A \mathbf{E})_{\epsilon}\right\|_{h} \leq c h^{r-1} \epsilon^{\left(-r+\frac{1}{2}\right)^{-}}\|A \mathbf{E}\|_{\mathbf{H}^{\frac{1}{2}}},
$$

provided $h<1$. For the second term, we use the error estimates (2.12)-(2.11) to obtain

$$
\left\|(A \mathbf{E})_{\epsilon}-A \mathbf{E}\right\|_{h} \leq c \epsilon^{\frac{1}{2}^{-}}\|\nabla \times A \mathbf{E}\|_{\mathbf{H}^{\frac{1}{2}^{-}}}+c\left(\epsilon^{\left(\alpha-\frac{1}{2}\right)^{-}}+h^{\alpha-1} \epsilon^{\frac{1^{-}}{2}}\right)\|A \mathbf{E}\|_{\mathbf{H}^{\frac{1}{2}}} .
$$

Finally, after gathering the above estimates we conclude that for $h<1$,

$$
\left\|A \mathbf{E}-A_{h} \mathbf{E}\right\|_{\mathbf{L}^{2}} \leq c\left(\epsilon^{\frac{1}{2}}+h^{\frac{1}{2}}+h^{r-1} \epsilon^{\left(-r+\frac{1}{2}\right)^{-}}+\epsilon^{\frac{1}{2}^{-}}+\epsilon^{\left(\alpha-\frac{1}{2}\right)^{-}}+h^{\alpha-1} \epsilon^{\frac{1}{2}^{-}}\right)\|\mathbf{E}\|_{\mathbf{L}^{2}} .
$$

The two dominating terms in the right-hand side are $h^{r-1} \epsilon^{\left(-r+\frac{1}{2}\right)^{-}}$and $h^{\alpha-1} \epsilon^{\frac{1}{2}^{-}}$, so that choosing $\epsilon=h^{\frac{r-\alpha}{r}}$ yields (4.7) for $h<1$. This estimate proves the pointwise convergence since $\alpha-\frac{1}{2}-\frac{\alpha}{2 r}>0$, owning to the restriction $\alpha \in\left(\frac{r}{2 r-1}, 1\right]$.

Remark 4.1 (Non-optimal Restriction on $\alpha$ ). The restriction on $\alpha$ in the above lemma is stronger than in Lemma 3.3. namely $\alpha>\frac{r}{2 r-1}$ instead of $\alpha>\frac{1}{2}$, and the error estimate (4.7) is slightly weaker than (3.11) since $\left(\left(\alpha-\frac{1}{2}\right) \frac{r-1}{\alpha+r-1}\right)^{-}>$ $\left(\alpha-\frac{1}{2}-\frac{\alpha}{2 r}\right)^{-}$for all $\alpha \in(0,1)$. We do not know whether this restriction on $\alpha$ and this loss of convergence rate are sharp, but they seem a reasonable price to pay for substituting the computation of the $H^{-\alpha}$-norm by the cheaper $H^{-1}$-norm. Finally, note that the lower bound on $\alpha$, i.e., $\frac{r}{2 r-1}$, converges to the optimal bound $\frac{1}{2}$ and the two convergence rates converge to $\left(\alpha-\frac{1}{2}\right)^{-}$as the polynomial order of the approximation, $r-1$, becomes large.

Remark 4.2 (Improvements). The estimates (3.11) and (4.7) can be improved whenever the regularity of $A \mathbf{E}$ can be a priori inferred to be better than that of $\mathbf{H}^{\frac{1}{2}}(\Omega)$. For instance, in two space dimensions the regularity of $A \mathbf{E}$ in a polygon depends on the angles at the vertices of $\Omega$. Estimates similar to (4.7) can also be obtained on $\left\|\nabla \times\left(A \mathbf{E}-A_{h} \mathbf{E}\right)\right\|_{\mathbf{L}^{2}}$. We refer to [22, 32 for more results in this direction.

Lemma 4.3 (Collective Compactness). Let $\alpha \in\left(\frac{1}{2}, 1\right)$. The sequence $\left\{A_{h}\right\}_{h>0}$ is collectively compact.

Proof. The proof is omitted since it is the same as that of Lemma 3.4 after replacing $\|\cdot\|$ by $\|\cdot\|_{h}$. 
As in the previous section the convergence of the eigenvalue problem cannot be deduced since the pointwise convergence only holds for solenoidal fields, i.e., $\mathbf{E} \in \mathbf{H}(\operatorname{div}=0, \Omega)$. The mixed method introduced in the next section resolves this is issue.

\section{MiXed And RElaxed $H^{-\alpha}$ Penalty}

To make the method discussed in $\$ 3$ and $\$ 4$ fully consistent and to make the relaxed penalty introduced in $\$ 4$ easier to implement in this section we introduce a mixed formulation. The use of a Lagrange multiplier will enable us to enforce the divergence free constraint and to construct an approximation of the $H^{-1}$-scalar product. The full consistency will lead to full pointwise convergence which in turn will imply spectral correctness, (compare Lemma 5.4 to Lemmas 3.3 and 4.2 ).

5.1. Formulation of the mixed-relaxed $H^{-\alpha}$ penalty. Consider the Laplace operator $-\Delta_{D}: H_{0}^{1}(\Omega) \longrightarrow H^{-1}(\Omega)$ associated with zero Dirichlet boundary condition. Recall that we defined in (2.1) the scalar product in $H^{-1}(\Omega)$ to be $\left\langle\cdot,\left(-\Delta_{D}\right)^{-1} \cdot\right\rangle$ so that $\|f\|_{H^{-1}}:=\left\langle f,\left(-\Delta_{D}\right)^{-1} f\right\rangle^{1 / 2}$.

Let $\mathbf{G}$ be an arbitrary vector field in $\mathbf{L}^{2}(\Omega)$, and let $p(\mathbf{G}) \in H_{0}^{1}(\Omega)$ so that

$$
\Delta p(\mathbf{G})=h^{2(\alpha-1)} \nabla \cdot \mathbf{G},\left.\quad p\right|_{\partial \Omega}=0 .
$$

Then, the following identity holds:

$$
h^{2(\alpha-1)}\langle\nabla \cdot \mathbf{F}, \nabla \cdot \mathbf{G}\rangle_{H^{-1}}=(\nabla p(\mathbf{G}), \mathbf{F}), \quad \forall \mathbf{F} \in \mathbf{L}^{2}(\Omega) .
$$

This observation implies that the bilinear form $a_{h}$ defined in (4.2) can then be rewritten as follows:

$$
a_{h}\left(\mathbf{E}_{h}, \mathbf{F}_{h}\right)=\left(\nabla \times \mathbf{E}_{h}, \nabla \times \mathbf{F}_{h}\right)+\left(\nabla p\left(\mathbf{E}_{h}\right), \mathbf{F}_{h}\right),
$$

where $p\left(\mathbf{E}_{h}\right)$ in $H_{0}^{1}(\Omega)$ is the function of $\nabla \cdot \mathbf{E}_{h}$ which solves the following problem: $\left(\nabla p\left(\mathbf{E}_{h}\right), \nabla q\right)=h^{2(\alpha-1)}\left(\mathbf{E}_{h}, \nabla q\right)$ for all $q$ in $H_{0}^{1}(\Omega)$. In the rest of this section we propose to replace the exact $H^{-1}$-scalar product by an approximate one by seeking the Lagrange multiplier $p$ in the approximation space $M_{h}$.

After replacing $H_{0}^{1}(\Omega)$ by the finite-dimensional space $M_{h}$, we are lead to consider the following discrete eigenvalue problem: Seek a triplet $\left(\lambda_{h} ; \mathbf{E}_{h}, p_{h}\right) \in \mathbb{R} \times$ $\mathbf{X}_{h} \backslash\{0\} \times M_{h}$ so that for all $\mathbf{F}_{h} \in \mathbf{X}_{h}$ and $q_{h} \in M_{h}$,

$$
\left\{\begin{array}{l}
\left(\nabla \times \mathbf{E}_{h}, \nabla \times \mathbf{F}_{h}\right)+\left(\nabla p_{h}, \mathbf{F}_{h}\right)=\lambda_{h}\left(\mathbf{E}_{h}, \mathbf{F}_{h}\right), \\
-\left(\mathbf{E}_{h}, \nabla q_{h}\right)+h^{2(1-\alpha)}\left(\nabla p_{h}, \nabla q_{h}\right)=0 .
\end{array}\right.
$$

It turns out that this formulation of the eigenvalue problem requires the pair $\left(\mathbf{X}_{h}, M_{h}\right)$ to satisfy a non-trivial compatibility condition to be convergent. To avoid this technicality, we consider instead the following alternative formulation: Seek a triplet $\left(\lambda_{h} ; \mathbf{E}_{h}, p_{h}\right) \in \mathbb{R} \times \mathbf{X}_{h} \backslash\{0\} \times M_{h}$ so that for all $\mathbf{F}_{h} \in \mathbf{X}_{h}$ and $q_{h} \in M_{h}$,

$$
\left\{\begin{array}{l}
\left(\nabla \times \mathbf{E}_{h}, \nabla \times \mathbf{F}_{h}\right)+\left(\nabla p_{h}, \mathbf{F}_{h}\right)+h^{2 \alpha}\left(\nabla \cdot \mathbf{E}_{h}, \nabla \cdot \mathbf{F}_{h}\right)=\lambda_{h}\left(\mathbf{E}_{h}, \mathbf{F}_{h}\right), \\
-\left(\mathbf{E}_{h}, \nabla q_{h}\right)+h^{2(1-\alpha)}\left(\nabla p_{h}, \nabla q_{h}\right)=0 .
\end{array}\right.
$$

Before dwelling on well-posedness of the above eigenvalue problem we provide the following lemma to justify the presence of the additional terms $h^{2 \alpha}\left(\nabla \cdot \mathbf{E}_{h}, \nabla \cdot \mathbf{F}_{h}\right)$. 
Lemma 5.1 (Discrete Control of $\nabla \cdot \mathbf{F}_{h}$ in $H^{-\alpha}(\Omega)$ ). Let $\alpha \in(0,1)$. There exists $c$ uniform in $h$, so that for $\mathbf{F}_{h} \in \mathbf{X}_{h}$,

$$
c\left\|\nabla \cdot \mathbf{F}_{h}\right\|_{H^{-\alpha}} \leq \sup _{0 \neq q_{h} \in M_{h}} \frac{\left(\mathbf{F}_{h}, \nabla q_{h}\right)}{h^{1-\alpha}\left\|\nabla q_{h}\right\|_{\mathbf{L}^{2}}}+h^{\alpha}\left\|\nabla \cdot \mathbf{F}_{h}\right\|_{L^{2}} .
$$

Proof. This is a standard perturbation argument. Owing to (2.14) (2.15), we have

$$
\begin{aligned}
\left\|\nabla \cdot \mathbf{F}_{h}\right\|_{H^{-\alpha}} & =\sup _{0 \neq q \in H_{0}^{\alpha}} \frac{\left(\nabla \cdot \mathbf{F}_{h}, q\right)}{\|q\|_{H^{\alpha}}} \\
& \leq \sup _{0 \neq q \in H_{0}^{\alpha}} \frac{\left(\nabla \cdot \mathbf{F}_{h}, q-\mathcal{C}_{h} q\right)}{\|q\|_{H^{\alpha}}}+\sup _{0 \neq q \in H_{0}^{\alpha}} \frac{\left(\nabla \cdot \mathbf{F}_{h}, \mathcal{C}_{h} q\right)}{\|q\|_{H^{\alpha}}} \\
& \leq \operatorname{ch}^{\alpha}\left\|\nabla \cdot \mathbf{F}_{h}\right\|_{L^{2}}+c \sup _{0 \neq q \in H_{0}^{\alpha}} \frac{\left(\nabla \cdot \mathbf{F}_{h}, \mathcal{C}_{h} q\right)}{\left\|\mathcal{C}_{h} q\right\|_{H^{\alpha}}} .
\end{aligned}
$$

The conclusion follows by using the inverse estimate $\left\|\nabla \mathcal{C}_{h} q\right\|_{\mathbf{L}^{2}} \leq c h^{\alpha-1}\left\|\mathcal{C}_{h} q\right\|_{H^{\alpha}}$.

To rewrite (5.2) in a more compact way we now define the bilinear form

$$
\begin{aligned}
d_{h}((\mathbf{E}, p),(\mathbf{F}, q))=(\nabla \times \mathbf{E}, \nabla \times \mathbf{F}) & +h^{2 \alpha}(\nabla \cdot \mathbf{E}, \nabla \cdot \mathbf{F}) \\
& +(\nabla p, \mathbf{F})-(\mathbf{E}, \nabla q)+h^{2(1-\alpha)}(\nabla p, \nabla q) .
\end{aligned}
$$

Then (5.2) is recast as follows: Seek a triplet $\left(\lambda_{h} ; \mathbf{E}_{h}, p_{h}\right) \in \mathbb{R} \times \mathbf{X}_{h} \backslash\{0\} \times M_{h}$ so that for all $\mathbf{F}_{h} \in \mathbf{X}_{h}$ and $q_{h} \in M_{h}$,

$$
d_{h}\left(\left(\mathbf{E}_{h}, p_{h}\right),\left(\mathbf{F}_{h}, q_{h}\right)\right)=\lambda_{h}\left(\mathbf{E}_{h}, \mathbf{F}_{h}\right), \quad \forall\left(\mathbf{F}_{h}, q_{h}\right) \in \mathbf{X}_{h} \times M_{h} .
$$

Let us define the following discrete norm:

$$
\|(\mathbf{E}, p)\|_{h}:=\|\mathbf{E}\|_{\mathbf{H}^{1-\alpha}}+h^{\alpha}\|\nabla \cdot \mathbf{E}\|_{L^{2}}+\|\nabla \times \mathbf{E}\|_{\mathbf{L}^{2}}+h^{1-\alpha}\|\nabla p\|_{\mathbf{L}^{2}} .
$$

The following result characterizes the stability of $d_{h}$.

Lemma 5.2 (Stability). For any $\alpha \in\left(\frac{1}{2}, 1\right)$ there is $c$, uniform in $h$, so that for all $\left(\mathbf{E}_{h}, p_{h}\right) \in \mathbf{X}_{h} \times M_{h}$ there holds

$$
\sup _{0 \neq\left(\mathbf{F}_{h}, q_{h}\right) \in \mathbf{X}_{h} \times M_{h}} \frac{d_{h}\left(\left(\mathbf{E}_{h}, p_{h}\right),\left(\mathbf{F}_{h}, q_{h}\right)\right)}{\left\|\left(\mathbf{F}_{h}, q_{h}\right)\right\|_{h}} \geq c\left\|\left(\mathbf{E}_{h}, p_{h}\right)\right\|_{h} .
$$

Proof. Let $\left(\mathbf{E}_{h}, p_{h}\right)$ be a non-zero member of $\mathbf{X}_{h} \times M_{h}$. Observe first that

$$
d_{h}\left(\left(\mathbf{E}_{h}, p_{h}\right),\left(\mathbf{E}_{h}, p_{h}\right)\right)=h^{2 \alpha}\left\|\nabla \cdot \mathbf{E}_{h}\right\|_{L^{2}}^{2}+\left\|\nabla \times \mathbf{E}_{h}\right\|_{\mathbf{L}^{2}}^{2}+h^{2(1-\alpha)}\left\|\nabla p_{h}\right\|_{\mathbf{L}^{2}}^{2} .
$$

Let us denote $\mathbb{S}$ the left-hand side in (5.7), then

$$
\mathbb{S}\left\|\left(\mathbf{E}_{h}, p_{h}\right)\right\|_{h} \geq h^{2 \alpha}\left\|\nabla \cdot \mathbf{E}_{h}\right\|_{L^{2}}^{2}+\left\|\nabla \times \mathbf{E}_{h}\right\|_{\mathbf{L}^{2}}^{2}+h^{2(1-\alpha)}\left\|\nabla p_{h}\right\|_{\mathbf{L}^{2}}^{2} .
$$

Observe also that for all $q_{h} \in M_{h}$ we have

$$
d_{h}\left(\left(\mathbf{E}_{h}, p_{h}\right),\left(0,-q_{h}\right)\right)=\left(\mathbf{E}_{h}, \nabla q_{h}\right)-h^{2(1-\alpha)}\left(\nabla p_{h}, \nabla q_{h}\right) .
$$

Then, assuming that $0 \neq q_{h}$, we deduce

$$
\begin{aligned}
\frac{d_{h}\left(\left(\mathbf{E}_{h}, p_{h}\right),\left(0,-q_{h}\right)\right)}{\left\|\left(0, q_{h}\right)\right\|_{h}} & \geq \frac{\left(\mathbf{E}_{h}, \nabla q_{h}\right)}{h^{1-\alpha}\left\|\nabla q_{h}\right\|_{\mathbf{L}^{2}}}-h^{1-\alpha}\left\|\nabla p_{h}\right\|_{\mathbf{L}^{2}} \\
& \geq \frac{\left(\mathbf{E}_{h}, \nabla q_{h}\right)}{h^{1-\alpha}\left\|\nabla q_{h}\right\|_{\mathbf{L}^{2}}}-\mathbb{S}^{\frac{1}{2}}\left\|\left(\mathbf{E}_{h}, p_{h}\right)\right\|_{h}^{\frac{1}{2}} .
\end{aligned}
$$


Taking the supremum over $q_{h}$ and using (5.3) from Lemma 5.1, we obtain

$$
\begin{aligned}
\mathbb{S} & \geq c\left\|\nabla \cdot \mathbf{E}_{h}\right\|_{H^{-\alpha}}-h^{\alpha}\left\|\nabla \cdot \mathbf{E}_{h}\right\|_{L^{2}}-\mathbb{S}^{\frac{1}{2}}\left\|\left(\mathbf{E}_{h}, p_{h}\right)\right\|_{h}^{\frac{1}{2}} \\
& \geq c\left\|\nabla \cdot \mathbf{E}_{h}\right\|_{H^{-\alpha}}-2 \mathbb{S}^{\frac{1}{2}}\left\|\left(\mathbf{E}_{h}, p_{h}\right)\right\|_{h}^{\frac{1}{2}} .
\end{aligned}
$$

As a result,

$$
\left\|\nabla \cdot \mathbf{E}_{h}\right\|_{H^{-\alpha}} \leq c\left(\mathbb{S}+\mathbb{S}^{\frac{1}{2}}\left\|\left(\mathbf{E}_{h}, p_{h}\right)\right\|_{h}^{\frac{1}{2}}\right) .
$$

Then recalling that $\mathbf{E}_{h} \times\left.\mathbf{n}\right|_{\partial \Omega}=0$ and using (3.4) from Lemma 3.1. we infer that

$$
c\left\|\mathbf{E}_{h}\right\|_{\mathbf{H}^{1-\alpha}} \leq\left\|\nabla \times \mathbf{E}_{h}\right\|_{\mathbf{L}^{2}}+\left\|\nabla \cdot \mathbf{E}_{h}\right\|_{H^{-\alpha}} \leq c\left(\mathbb{S}+\mathbb{S}^{\frac{1}{2}}\left\|\left(\mathbf{E}_{h}, p_{h}\right)\right\|_{h}^{\frac{1}{2}}\right) .
$$

Combining the above estimates, we finally obtain

$$
\left\|\left(\mathbf{E}_{h}, p_{h}\right)\right\|_{h} \leq c\left(\mathbb{S}+\mathbb{S}^{\frac{1}{2}}\left\|\left(\mathbf{E}_{h}, p_{h}\right)\right\|_{h}^{\frac{1}{2}}\right) \leq c \mathbb{S}+\frac{1}{2}\left\|\left(\mathbf{E}_{h}, p_{h}\right)\right\|_{h} .
$$

The conclusion follows readily.

Again, abusing the notation, we now redefine the discrete self-adjoint operator $A_{h}: \mathbf{L}^{2}(\Omega) \rightarrow \mathbf{X}_{h}: \mathbf{E} \mapsto A_{h} \mathbf{E}$ so that $A_{h} \mathbf{E}$ is the solution to

$$
d_{h}\left(\left(A_{h} \mathbf{E}, p_{h}\right),\left(\mathbf{F}_{h}, q_{h}\right)\right)=\left(\mathbf{E}, \mathbf{F}_{h}\right), \quad \forall\left(\mathbf{F}_{h}, q_{h}\right) \in \mathbf{X}_{h} \times M_{h} .
$$

Owing to the BNB theorem (see e.g. [24, Thm 2.6]), the inf-sup condition (5.7) guarantees the existence and uniqueness of the pair $\left(A_{h} \mathbf{E}, p_{h}\right)$; see also [24, Thm 2.22]. Finally, observe that $\left(\mathbf{E}_{h}, \lambda_{h}\right) \in \mathbf{X}_{h} \backslash\{0\} \times \mathbb{R} \backslash\{0\}$ is an eigenpair of (5.2) if and only if it satisfies

$$
A_{h} \mathbf{E}_{h}=\frac{1}{\lambda_{h}} \mathbf{E}_{h}
$$

Lemma 5.3 (Consistency). For any $\mathbf{E} \in \mathbf{L}^{2}(\Omega)$, the pair $\left(A_{h} \mathbf{E}, p_{h}\right)$ defined in (5.8) satisfies the following consistency relation:

$(5.9) d_{h}\left(\left(A \mathbf{E}-A_{h} \mathbf{E}, p-p_{h}\right),\left(\mathbf{F}_{h}, q_{h}\right)\right)=h^{2(1-\alpha)}\left(\nabla p, \nabla q_{h}\right), \quad \forall\left(\mathbf{F}_{h}, q_{h}\right) \in \mathbf{X}_{h} \times M_{h}$, where $p \in H_{0}^{1}(\Omega)$ is such that $\nabla \times \nabla \times A \mathbf{E}+\nabla p=\mathbf{E}$.

Proof. The definition of $A \mathbf{E}$ implies that there is $p \in H_{0}^{1}(\Omega)$ so that $\nabla \times \nabla \times A \mathbf{E}+$ $\nabla p=\mathbf{E}$; see (2.4). Since $\nabla \cdot A \mathbf{E}=0$ and $M_{h}$ is conforming in $H_{0}^{1}(\Omega)$, we have

$$
\begin{aligned}
d_{h}\left((A \mathbf{E}, p),\left(\mathbf{F}_{h}, q_{h}\right)\right) & =\left(\nabla \times A \mathbf{E}, \nabla \times \mathbf{F}_{h}\right)+\left(\nabla p, \mathbf{F}_{h}\right)+h^{2(1-\alpha)}\left(\nabla p, \nabla q_{h}\right) \\
& =\left(\mathbf{E}, \mathbf{F}_{h}\right)+h^{2(1-\alpha)}\left(\nabla p, \nabla q_{h}\right) \\
& =d_{h}\left(\left(A_{h} \mathbf{E}, p_{h}\right),\left(\mathbf{F}_{h}, q_{h}\right)\right)+h^{2(1-\alpha)}\left(\nabla p, \nabla q_{h}\right),
\end{aligned}
$$

which proves the statement.

5.2. Convergence of mixed-relaxed $H^{-\alpha}$ penalty. The convergence analysis is done by proving the pointwise convergence and the collective compactness property.

Lemma 5.4 (Pointwise Convergence). Let $r \geq 2$ be the restriction on the approximation estimate (2.15) and let $\alpha \in\left(\frac{r}{2 r-1}, 1\right]$. Then the sequence $\left\{A_{h}\right\}_{h>0}$ converges pointwise to A. More precisely, there exists a constant $c$ independent of the mesh size $h$ such that

$$
\left\|A \mathbf{E}-A_{h} \mathbf{E}\right\|_{\mathbf{L}^{2}} \leq c\left(h^{\left(\alpha-\frac{1}{2}-\frac{\alpha}{2 r}\right)^{-}}\|\mathbf{E}\|_{\mathbf{L}^{2}}+h^{1-\alpha}\|\nabla \cdot \mathbf{E}\|_{H^{-1}(\Omega)}\right), \forall 0<h<1 .
$$


In particular, for the optimal choice $\alpha=\frac{3 r}{4 r-1}$ we have

$$
\left\|A \mathbf{E}-A_{h} \mathbf{E}\right\|_{\mathbf{L}^{2}} \leq c h^{\left(\frac{r-1}{4 r-1}\right)^{-}}\|\mathbf{E}\|_{\mathbf{L}^{2}}, \quad \forall 0<h<1,
$$

and the following holds whenever $\nabla \cdot \mathbf{E}=0$ :

$$
\left\|A \mathbf{E}-A_{h} \mathbf{E}\right\|_{\mathbf{L}^{2}} \leq c h^{\left(\alpha-\frac{1}{2}-\frac{\alpha}{2 r}\right)^{-}}\|\mathbf{E}\|_{\mathbf{L}^{2}(\Omega)} .
$$

Proof. The proof is again similar to that of Lemma 3.3, and by repeating the same arguments we have

$$
\begin{aligned}
\left\|A \mathbf{E}-A_{h} \mathbf{E}\right\|_{\mathbf{L}^{2}} & \leq\left\|A \mathbf{E}-(A \mathbf{E})_{\epsilon}\right\|_{\mathbf{L}^{2}}+\left\|(A \mathbf{E})_{\epsilon}-\mathcal{C}_{h}(A \mathbf{E})_{\epsilon}\right\|_{\mathbf{L}^{2}}+\left\|\mathcal{C}_{h}(A \mathbf{E})_{\epsilon}-A_{h} \mathbf{E}\right\|_{\mathbf{L}^{2}} \\
& \leq c\left(h^{\frac{1}{2}}+\epsilon^{\frac{1}{2}}\right)\|\mathbf{E}\|_{\mathbf{L}^{2}}+\left\|\mathcal{C}_{h}(A \mathbf{E})_{\epsilon}-A_{h} \mathbf{E}\right\|_{\mathbf{L}^{2}} .
\end{aligned}
$$

The rest of the proof consists of deriving an estimate for $\left\|\mathcal{C}_{h}(A \mathbf{E})_{\epsilon}-A_{h} \mathbf{E}\right\|_{\mathbf{L}^{2}}$.

Let $p_{h} \in M_{h}$ be the Lagrange multiplier associated with $A \mathbf{E}_{h}$ in (5.8). The inf-sup condition (5.2) and the consistency (5.9) imply

$$
\begin{aligned}
& c\left\|\mathcal{C}_{h}(A \mathbf{E})_{\epsilon}-A_{h} \mathbf{E}\right\|_{\mathbf{L}^{2}(\Omega)} \leq c\left\|\left(\mathcal{C}_{h}(A \mathbf{E})_{\epsilon}-A_{h} \mathbf{E}, \mathcal{C}_{h} p-p_{h}\right)\right\|_{h} \\
& \leq \sup _{0 \neq\left(\mathbf{F}_{h}, q_{h}\right) \in \mathbf{X}_{h} \times M_{h}} \frac{d_{h}\left(\left(\mathcal{C}_{h}(A \mathbf{E})_{\epsilon}-A_{h} \mathbf{E}, \mathcal{C}_{h} p-p_{h}\right),\left(\mathbf{F}_{h}, q_{h}\right)\right)}{\left\|\mid\left(\mathbf{F}_{h}, q_{h}\right)\right\| \|_{h}} \\
& \leq \sup _{0 \neq\left(\mathbf{F}_{h}, q_{h}\right) \in \mathbf{X}_{h} \times M_{h}} \frac{d_{h}\left(\left(\mathcal{C}_{h}(A \mathbf{E})_{\epsilon}-A \mathbf{E}, \mathcal{C}_{h} p-p\right),\left(\mathbf{F}_{h}, q_{h}\right)\right)}{\left\|\left(\mathbf{F}_{h}, q_{h}\right)\right\| \|_{h}} \\
& \quad+\sup _{0 \neq q_{h} \in M_{h}} h^{2(1-\alpha)} \frac{\left(\nabla p, \nabla q_{h}\right)}{\left\|\left(0, q_{h}\right)\right\| \|_{h}} \\
& \leq\left\|\nabla \times\left(A \mathbf{E}-\mathcal{C}_{h}(A \mathbf{E})_{\epsilon}\right)\right\|_{\mathbf{L}^{2}}+h^{\alpha}\left\|\nabla \cdot\left(A \mathbf{E}-\mathcal{C}_{h}(A \mathbf{E})_{\epsilon}\right)\right\|_{L^{2}} \\
& \quad+h^{\alpha-1}\left\|A \mathbf{E}-\mathcal{C}_{h}(A \mathbf{E})_{\epsilon}\right\|_{\mathbf{L}^{2}} \\
& \quad+h^{1-\alpha}\left\|\nabla\left(\mathcal{C}_{h} p-p\right)\right\|_{\mathbf{L}^{2}}+h^{-\alpha}\left\|\mathcal{C}_{h} p-p\right\|_{L^{2}}+h^{1-\alpha}\|\nabla p\|_{\mathbf{L}^{2}} .
\end{aligned}
$$

We now bound separately the terms appearing on the right-hand side of the above estimate.

For the first term, we invoke (2.12), the approximation estimates (2.15) with $t=1, l=r$, and (2.13) with $s=r$. We obtain

$$
\begin{aligned}
\left\|\nabla \times\left(A \mathbf{E}-\mathcal{C}_{h}(A \mathbf{E})_{\epsilon}\right)\right\|_{\mathbf{L}^{2}} & \leq\left\|\nabla \times\left(A \mathbf{E}-(A \mathbf{E})_{\epsilon}\right)\right\|_{\mathbf{L}^{2}}+\left\|\nabla \times\left((A \mathbf{E})_{\epsilon}-\mathcal{C}_{h}(A \mathbf{E})_{\epsilon}\right)\right\|_{\mathbf{L}^{2}} \\
& \leq c_{1}{\epsilon^{\frac{1}{2}}}^{-}\|\nabla \times A \mathbf{E}\|_{\mathbf{H}^{\frac{1}{2}}}+c_{2} h^{r-1} \epsilon^{\left(-r+\frac{1}{2}\right)^{-}}\|A \mathbf{E}\|_{\mathbf{H}^{\frac{1}{2}}} .
\end{aligned}
$$

For the second term we apply the approximation estimate (2.15) with $t=1$, $l=r,(2.13)$ with $s=r$, and the inverse estimate (2.13) with $s=1$ to obtain

$$
\begin{aligned}
h^{\alpha}\left\|\nabla \cdot\left(A \mathbf{E}-\mathcal{C}_{h}(A \mathbf{E})_{\epsilon}\right)\right\|_{L^{2}} & \leq h^{\alpha}\left\|\nabla \cdot(A \mathbf{E})_{\epsilon}\right\|_{L^{2}}+h^{\alpha}\left\|(A \mathbf{E})_{\epsilon}-\mathcal{C}_{h}(A \mathbf{E})_{\epsilon}\right\|_{\mathbf{H}^{1}} \\
& \leq c_{1} h^{\alpha}\left\|(A \mathbf{E})_{\epsilon}\right\|_{\mathbf{H}^{1}}+c_{2} h^{\alpha+r-1} \epsilon^{\left(-r+\frac{1}{2}\right)^{-}}\|A \mathbf{E}\|_{\mathbf{H}^{\frac{1}{2}}} \\
& \leq c\left(h^{\alpha} \epsilon^{\left(-\frac{1}{2}\right)^{-}}+h^{\alpha+r-1} \epsilon^{\left(-r+\frac{1}{2}\right)^{-}}\right)\|A \mathbf{E}\|_{\mathbf{H}^{\frac{1}{2}}} .
\end{aligned}
$$

Note that we used the property $\nabla \cdot A \mathbf{E}=0$ in the first inequality. 
Similarly, for the third term invoking (2.11) with $s=0$ and the approximation estimate (2.15) with $t=0, l=r$ coupled with (2.13) with $s=r$ we arrive at

$$
\begin{aligned}
h^{\alpha-1}\left\|A \mathbf{E}-\mathcal{C}_{h}(A \mathbf{E})_{\epsilon}\right\|_{\mathbf{L}^{2}} & \leq h^{\alpha-1}\left\|A \mathbf{E}-(A \mathbf{E})_{\epsilon}\right\|_{\mathbf{L}^{2}}+h^{\alpha-1}\left\|(A \mathbf{E})_{\epsilon}-\mathcal{C}_{h}(A \mathbf{E})_{\epsilon}\right\|_{\mathbf{L}^{2}} \\
& \leq c\left(h^{\alpha-1} \epsilon^{\frac{1}{2}^{-}}+h^{\alpha+r-1} \epsilon^{\left(-r+\frac{1}{2}^{-}\right)}\right)\|A \mathbf{E}\|_{\mathbf{H}^{\frac{1}{2}}} .
\end{aligned}
$$

The last three terms involving $p$ are bounded as follows:

$$
\begin{aligned}
h^{1-\alpha}\left\|\nabla\left(\mathcal{C}_{h} p-p\right)\right\|_{\mathbf{L}^{2}}+h^{-\alpha}\left\|\mathcal{C}_{h} p-p\right\|_{L^{2}} & +h^{1-\alpha}\|\nabla p\|_{\mathbf{L}^{2}} \\
& \leq c h^{1-\alpha}\|\nabla p\|_{\mathbf{L}^{2}} \leq c h^{1-\alpha}\|\nabla \cdot \mathbf{E}\|_{H^{-1}},
\end{aligned}
$$

where we used the estimate on the pressure (2.5).

By gathering the above estimates we obtain

$$
\begin{aligned}
\left\|A \mathbf{E}-A_{h} \mathbf{E}\right\|_{\mathbf{L}^{2}} \leq c & \left(h^{\frac{1}{2}^{2}}+\epsilon^{\frac{1}{2}^{-}}+h^{r-1} \epsilon^{\left(-r+\frac{1}{2}\right)^{-}}+h^{\alpha} \epsilon^{\left(-\frac{1}{2}\right)^{-}}\right. \\
& \left.+h^{\alpha+r-1} \epsilon^{\left(-r+\frac{1}{2}\right)^{-}}+h^{\alpha-1} \epsilon^{\frac{1}{2}^{-}}\right)\|\mathbf{E}\|_{\mathbf{L}^{2}} \\
& +c h^{1-\alpha}\|\nabla \cdot \mathbf{E}\|_{H^{-1}} .
\end{aligned}
$$

The two dominating terms in the coefficient in front of $\|\mathbf{E}\|_{\mathbf{L}^{2}}$ are $h^{r-1} \epsilon^{\left(-r+\frac{1}{2}\right)^{-}}$ and $h^{\alpha-1} \epsilon^{\frac{1}{2}^{-}}$. Therefore, assuming $h \leq 1$ and choosing $\epsilon=h^{\frac{r-\alpha}{r}}$ implies (5.10). This estimate proves the pointwise convergence since $\alpha-\frac{1}{2}-\frac{\alpha}{2 r}>0$, owing to the restriction $\alpha \in\left(\frac{r}{2 r-1}, 1\right]$.

Remark 5.1 (Non-optimal Restriction on $\alpha$ ). Similarly, to the relaxed $H^{-\alpha}$ penalty technique (see Remark 4.1), the restriction $\alpha>\frac{r}{2 r-1}$ is not optimal; but quasioptimality is recovered in the limit $r \rightarrow \infty$. Similarly, the convergence rate (5.12) is quasi-optimal in the limit $r \rightarrow \infty$ for $\alpha=1$.

Lemma 5.5 (Collective Compactness). Let $\alpha \in\left(\frac{1}{2}, 1\right)$. The sequence $\left\{A_{h}\right\}_{h>0}$ is collectively compact.

Proof. Let $\mathbf{E}$ be a member of $\mathbf{L}^{2}(\Omega)$. Let $p_{h} \in M_{h}$ be the Lagrange multiplier associated with $A_{h} \mathbf{E}$ in (5.8). The inf-sup condition (5.7) together with the definition (5.8) of the operator $A_{h}$ and the Cauchy-Schwarz inequality imply that

$$
\begin{aligned}
c\left\|A_{h} \mathbf{E}\right\|_{\mathbf{H}^{1-\alpha}} & \leq c\left\|\left(A_{h} \mathbf{E}, p_{h}\right)\right\|_{h} \\
& \leq \sup _{0 \neq\left(\mathbf{F}_{h}, q_{h}\right) \in \mathbf{X}_{h} \times M_{h}} \frac{d_{h}\left(\left(A_{h} \mathbf{E}, p_{h}\right),\left(\mathbf{F}_{h}, q_{h}\right)\right)}{\left\|\mid\left(\mathbf{F}_{h}, q_{h}\right)\right\| \|_{h}} \\
& \leq \sup _{0 \neq\left(\mathbf{F}_{h}, q_{h}\right) \in \mathbf{X}_{h} \times M_{h}} \frac{\left(\mathbf{E}, \mathbf{F}_{h}\right)}{\left\|\left(\mathbf{F}_{h}, q_{h}\right)\right\|_{h}} \leq\|\mathbf{E}\|_{\mathbf{H}^{\alpha-1}} .
\end{aligned}
$$

We finish by invoking the same arguments as in the proof of Lemma 3.4.

Theorem 5.1 (Convergence). Let $\Omega$ be a bounded, simply connected, Lipschitz domain in $\mathbb{R}^{d}, d=2,3$. Let $r$ be the restriction on the approximation estimate (2.15) and $\alpha \in\left(\frac{r}{2 r-1}, 1\right)$. Then (5.2) is a spectrally correct approximation of (1.2) in the sense that the conclusions of Theorem 2.2 hold.

Proof. We apply Theorem 2.2. The pointwise convergence of the sequence $\left\{A_{h}\right\}_{h>0}$ to $A$ is proved in Lemma 5.4 and the collective compactness of the sequence is proved in Lemma 5.5 . 
Remark 5.2 (Choice of the Approximation Space $M_{h}$ for $p$ ). The spectral correctness stated in Theorem 5.1 is independent of the choice of $M_{h} \subset H_{0}^{1}(\Omega)$ provided that the inf-sup condition (5.7) holds; in particular, $\mathbb{P}_{1}$ Lagrange finite elements are sufficient for this purpose. But, of course, the convergence rate on the approximation of smooth eigenpairs depends on the approximation properties in $M_{h}$; higher convergence rates require better approximation properties in $M_{h}$.

\section{Numerical tests}

To illustrate the performance of the method described in this paper, we now present some finite element computations performed in the L-shape domain

$$
\Omega=(-1,+1)^{2} \backslash([0,+1] \times[-1,0]) .
$$

We start with the boundary value problem and then solve the eigenvalue problem. We observe that, as claimed in Theorem 5.1, the method is spectrally correct, i.e., there are no spurious eigenvalues and the approximate eigenvalues converge appropriately. We conclude by a discussion on the choice of the parameter $\alpha$ and on the imposition of the boundary conditions.

6.1. Boundary value problem. Consider the L-shape domain defined in (6.1), and let $\mathbf{E}$ be the solution to the following boundary value problem:

$$
\nabla \times \nabla \times \mathbf{E}=0, \quad \nabla \cdot \mathbf{E}=0, \quad \mathbf{E} \times\left.\mathbf{n}\right|_{\partial \Omega}=\mathbf{G} \times \mathbf{n},
$$

where the Cartesian components of the boundary data $\mathbf{G}$ are given by

$$
\mathbf{G}(r, \theta)=\frac{2}{3} r^{-\frac{1}{3}}\left(\begin{array}{r}
-\sin \left(\frac{\theta}{3}\right) \\
\cos \left(\frac{\theta}{3}\right)
\end{array}\right),
$$

and $(r, \theta)$ are the polar coordinates centered at the re-entrant corner of the domain. The solution to the above problem is $\mathbf{E}=\nabla \varphi$, where $\varphi(r, \theta)=r^{\frac{2}{3}} \sin \left(\frac{2}{3} \theta\right)$, and $\mathbf{E} \in \mathbf{H}^{\frac{2}{3}}(\Omega)$.

Five quasi-uniform (non-nested) Delaunay meshes are considered of mesh sizes $1 / 10,1 / 20,1 / 40,1 / 80,1 / 160$, respectively. The meshes are composed of triangles. Two types of approximation are tested; we use $\mathbb{P}_{1}$ elements in the first case and $\mathbb{P}_{2}$ elements in the second case. The electric field and the Lagrange multiplier are approximated using equal order polynomials in each case.

TABLE 1. $L^{2}(\Omega)$ relative errors for the boundary value problem (6.2)-(6.3) using $\mathbb{P}_{1}$ elements (2nd and 3rd columns) and $\mathbb{P}_{2}$ elements (4th \& 5th columns) with $\alpha=0.75$ and $\alpha=1$.

\begin{tabular}{|l||c|c|c|c||c|c|c|c|}
\hline \multirow{2}{*}{\multicolumn{1}{|c||}{$\mathrm{h}$}} & \multicolumn{4}{c||}{$\mathbb{P}_{1}$} & \multicolumn{4}{c|}{$\mathbb{P}_{2}$} \\
\cline { 2 - 9 } & \multicolumn{2}{|c|}{$\alpha=0.75$} & \multicolumn{2}{|c|}{$\alpha=1$} & \multicolumn{2}{c|}{$\alpha=0.75$} & \multicolumn{2}{c|}{$\alpha=1$} \\
\cline { 2 - 9 } & Rel. Error & COC & Rel. Error & COC & Rel. Error & COC & Rel. Error & COC \\
\hline 0.1 & $2.39010^{-1}$ & N/A & $2.30310^{-1}$ & N/A & $1.29010^{-1}$ & N/A & $1.11010^{-1}$ & N/A \\
0.05 & $1.84310^{-1}$ & 0.38 & $1.82610^{-1}$ & 0.34 & $8.17810^{-2}$ & 0.66 & $7.01610^{-2}$ & 0.66 \\
0.025 & $1.40510^{-1}$ & 0.39 & $1.36710^{-1}$ & 0.42 & $5.97810^{-2}$ & 0.45 & $5.01710^{-2}$ & 0.48 \\
0.0125 & $1.03110^{-1}$ & 0.45 & $1.01010^{-1}$ & 0.44 & $3.75910^{-2}$ & 0.67 & $3.19110^{-2}$ & 0.65 \\
0.00625 & $7.54410^{-2}$ & 0.45 & $7.65610^{-2}$ & 0.4 & $2.23210^{-2}$ & 0.75 & $1.93810^{-2}$ & 0.72 \\
\hline
\end{tabular}

The results are reported in Table 1, the relative errors in the $\mathbf{L}^{2}$-norm are shown for $\alpha=0.75$ and $\alpha=1$ together with the computed order of convergence (COC). Convergence is observed for the $\mathbb{P}_{1}$ and the $\mathbb{P}_{2}$ approximations. The observed 
convergence rates depend weakly on $\alpha$ and are clearly better than (5.10). Since $\mathbf{E} \in \mathbf{H}^{\frac{2}{3}}(\Omega)$ the best possible convergence rate is $\frac{2}{3}$ and this rate is achieved numerically when using $\mathbb{P}_{2}$ elements.

6.2. Eigenvalue problem. We now turn our attention to the eigenvalue problem in the L-shaped domain (6.1). Approximate values of the first five eigenvalues with $10^{-11}$ tolerance are provided in 23 : $\lambda_{1} \approx 1.47562182408, \lambda_{2} \approx 3.53403136678$, $\lambda_{3}=\lambda_{4}=\pi^{2} \approx 9.86960440109$, and $\lambda_{5} \approx 11.3894793979$. We use the same five quasi-uniform triangular meshes of mesh sizes $1 / 10,1 / 20,1 / 40,1 / 80,1 / 160$, as in 6.1. We use ARPACK [33] with a relative tolerance of $10^{-8}$ to obtain the approximation of the eigenvalue problem (5.2).

Two sets of results are presented below. First, we compute the first eigenvalue with $\alpha=0.9$. Second, we compute the first five eigenvalues with $\alpha=0.7$. We refer to 10 for comparable results using the mixed $L^{2}$-weighted method.

6.2.1. The first eigenvalue. The first eigenvalue is the most difficult to evaluate since it corresponds to the eigen-vector of lowest regularity in $\mathbf{H}^{\frac{2}{3}}(\Omega)$. The results for $\lambda_{1}$ using $\alpha=0.9$ are reported in Table 2, The method converges as expected. The convergence behavior of the method with respect to the mesh size $h$ is similar to what was observed for the boundary value problem. We observe a convergence rate close to first-order for the $\mathbb{P}_{2}$ approximation.

TABle 2. Relative errors and COC for $\lambda_{1}$ using $\mathbb{P}_{1}$ elements (2nd and 3rd columns) and $\mathbb{P}_{2}$ elements (4th and 5th columns) with $\alpha=0.9$. The symbol "-" indicates that the pair (Linear Solver + ARPACK) did not converge with the assigned tolerances.

\begin{tabular}{|l||c|c|c||c|c|c|}
\hline \multirow{2}{*}{\multicolumn{1}{|c||}{$\mathrm{h}$}} & \multicolumn{3}{c||}{$\mathbb{P}_{1}$} & \multicolumn{3}{c|}{$\mathbb{P}_{2}$} \\
\cline { 2 - 7 } & $\lambda_{1}$ & Rel. Error & COC & $\lambda_{1}$ & Rel. Error & COC \\
\hline 0.1 & 1.555 & $5.25610^{-2}$ & N/A & 1.508 & $2.19210^{-2}$ & N/A \\
0.05 & 1.541 & $4.35310^{-2}$ & 0.27 & 1.493 & $1.16710^{-2}$ & 0.9 \\
0.025 & 1.522 & $3.09410^{-2}$ & 0.49 & 1.487 & $7.37110^{-3}$ & 0.66 \\
0.0125 & 1.507 & $2.12610^{-2}$ & 0.54 & 1.481 & $3.72610^{-3}$ & 0.98 \\
0.00625 & 1.497 & $1.46510^{-2}$ & 0.54 & - & - & N/A \\
\hline
\end{tabular}

6.2.2. The first five eigenvalues. We now compute the first five eigenvalues with $\alpha=0.7$. The results are reported in Table 3 . No spurious eigenvalue is observed and convergence to the exact eigenvalues is obtained in accordance with Theorem 5.1. As expected, the worst rate of convergence is observed for the first eigenvalue which corresponds to the most singular eigenvector. A better COC is achieved for the first eigenvalue by increasing $\alpha$; see Section 6.2.1. Finally, we mention that the COC stalls for the eigenvalues $\lambda_{3}$ and $\lambda_{4}$ using $\mathbb{P}_{2}$ since the accuracy of the computed eigenvalues is limited by the tolerance in ARPACK $\left(10^{-8}\right)$.

6.3. Choice of $\alpha$. A second look at the proofs of the pointwise convergence (Lemma 5.4) and the collective compactness (Lemma 5.5) suggest that choosing $\alpha$ close to 1 increases the pointwise convergence rate while choosing $\alpha$ close to $\frac{1}{2}$ improves the collective compactness property. This fact is reflected in our numerical experiments. The COC for the first eigenvalue improves as $\alpha$ approaches 1 , 
TABLE 3. Relative errors and COC for the first five eigenvalues using $\mathbb{P}_{1}$ elements (left table) and $\mathbb{P}_{2}$ elements (right table) with $\alpha=0.7$. The symbol "-" indicates that the pair (Linear Solver + ARPACK) did not converge with the assigned tolerances.

\begin{tabular}{|c|c|c|c|c|c|c|}
\hline \multirow{2}{*}{$\mathrm{h}$} & \multicolumn{3}{|c|}{$\mathbb{P}_{1}$} & \multicolumn{3}{|c|}{$\mathbb{P}_{2}$} \\
\hline & $\lambda_{1}$ & Rel. Error & $\mathrm{COC}$ & $\lambda_{1}$ & Rel. Error & $\mathrm{COC}$ \\
\hline 0.1 & 1.930 & $2.66810^{-1}$ & $\mathrm{~N} / \mathrm{A}$ & 1.707 & $1.45210^{-1}$ & $\mathrm{~N} / \mathrm{A}$ \\
\hline 0.05 & 1.845 & $2.22410^{-1}$ & 0.26 & 1.623 & $9.52210^{-2}$ & 0.61 \\
\hline 0.025 & 1.765 & $1.78810^{-1}$ & 0.32 & 1.586 & $7.24010^{-2}$ & 0.4 \\
\hline 0.0125 & 1.696 & $1.38910^{-1}$ & 0.36 & 1.545 & $4.61410^{-2}$ & 0.65 \\
\hline 0.006256 & 1.644 & $1.08010^{-1}$ & 0.36 & 政 & - & $\mathrm{N} / \mathrm{A}$ \\
\hline \multirow{2}{*}{ h } & \multicolumn{3}{|c|}{$\overline{\mathbb{P}_{1}}$} & \multicolumn{3}{|c|}{$\overline{\mathbb{P}_{2}}$} \\
\hline & $\lambda_{2}$ & Rel. Error & $\mathrm{COC}$ & $\lambda_{2}$ & Rel. Error & $\mathrm{COC}$ \\
\hline 0.1 & 3.573 & $1.10110^{-2}$ & $\mathrm{~N} / \mathrm{A}$ & 3.537 & $8.26610^{-4}$ & $\mathrm{~N} / \mathrm{A}$ \\
\hline 0.05 & 3.551 & $4.71610^{-3}$ & 1.22 & 3.535 & $2.38010^{-4}$ & 1.8 \\
\hline 0.025 & 3.540 & $1.57810^{-3}$ & 1.58 & 3.534 & $6.64010^{-5}$ & 1.8 \\
\hline 0.0125 & 3.536 & $6.24510^{-4}$ & 1.33 & 3.534 & $1.72610^{-5}$ & 1.9 \\
\hline 0.006256 & 3.535 & $2.76810^{-4}$ & 1.17 & - & - & $\mathrm{N} / \mathrm{A}$ \\
\hline \multirow{2}{*}{ h } & \multicolumn{3}{|c|}{$\mathbb{P}_{1}$} & \multicolumn{3}{|c|}{$\mathbb{P}_{2}$} \\
\hline & $\lambda_{3}$ & Rel. Error & $\mathrm{COC}$ & $\lambda_{3}$ & Rel. Error & $\mathrm{COC}$ \\
\hline 0.1 & 5.450 & $5.77010^{-1}$ & $\mathrm{~N} / \mathrm{A}$ & 7.828 & $2.30710^{-1}$ & $\mathrm{~N} / \mathrm{A}$ \\
\hline 0.05 & 7.852 & $2.27710^{-1}$ & 1.34 & 9.870 & $3.79910^{-7}$ & 19.21 \\
\hline 0.025 & 9.873 & $3.07510^{-4}$ & 2.89 & 9.870 & $3.85610^{-8}$ & 3.3 \\
\hline 0.0125 & 9.870 & $7.71410^{-5}$ & 2.0 & 9.870 & $3.44410^{-8}$ & 0.16 \\
\hline 0.006256 & 9.870 & $1.93410^{-5}$ & 2.0 & 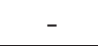 & - & $\mathrm{N} / \mathrm{A}$ \\
\hline \multirow{2}{*}{ h } & \multicolumn{3}{|c|}{$\mathbb{P}_{1}$} & \multicolumn{3}{|c|}{$\mathbb{P}_{2}$} \\
\hline & $\lambda_{4}$ & Rel. Error & $\mathrm{COC}$ & $\lambda_{4}$ & Rel. Error & $\mathrm{COC}$ \\
\hline 0.1 & 5.455 & $5.76110^{-1}$ & $\mathrm{~N} / \mathrm{A}$ & 7.841 & $2.29110^{-1}$ & $\mathrm{~N} / \mathrm{A}$ \\
\hline 0. & 7.858 & $2.27010^{-1}$ & 1.34 & 9.870 & $4.71210^{-7}$ & 18.9 \\
\hline 0.025 & 9.873 & $3.10010^{-4}$ & 9.52 & 9.870 & $3.85610^{-8}$ & 3.61 \\
\hline 0.0125 & 9.870 & $7.76810^{-5}$ & 2.0 & 9.870 & $1.99010^{-8}$ & 0.95 \\
\hline 0.00625 & 9.870 & $1.93510^{-5}$ & 2.0 & 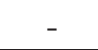 & - & $\mathrm{N} / \mathrm{A}$ \\
\hline \multirow{2}{*}{ h } & \multicolumn{3}{|c|}{$\mathbb{P}_{1}$} & \multicolumn{3}{|c|}{$\overline{\mathbb{P}_{2}}$} \\
\hline & $\lambda_{5}$ & Rel. Error & $\mathrm{COC}$ & $\lambda_{5}$ & Rel. Error & $\mathrm{COC}$ \\
\hline 0.1 & 5.506 & $6.96410^{-1}$ & $\mathrm{~N} / \mathrm{A}$ & 7.903 & $3.61410^{-1}$ & $\mathrm{~N} / \mathrm{A}$ \\
\hline 0.05 & 7.877 & $3.64610^{-1}$ & 0.93 & 11.39 & $2.37410^{-5}$ & 13.89 \\
\hline 0.025 & 11.39 & $4.32610^{-4}$ & 9.72 & 11.39 & $7.78610^{-6}$ & 1.61 \\
\hline 0.01 & 11.39 & $1.45710^{-4}$ & 1.57 & 11.39 & $2.16810^{-6}$ & 1.85 \\
\hline 0.006256 & 11.39 & $5.30310^{-5}$ & 1.46 & - & - & $\mathrm{N} / \mathrm{A}$ \\
\hline
\end{tabular}

but then the mesh size threshold $h_{0}$, so that the asymptotic convergence regime is observed for all $h \leq h_{0}$, decreases for the other eigenvalues. When $\alpha$ is close to $\frac{1}{2}$ we observe a deterioration on the COC for the first eigenvalue but the mesh size threshold $h_{0}$ for the asymptotic convergence regime on the others improves. This effect can be reduced by playing with an additional coefficient multiplying the 
stabilization term $h^{2 \alpha}\left(\nabla \cdot \mathbf{E}_{h}, \nabla \cdot \mathbf{F}_{h}\right)$ in formulation (5.2). This tuning possibility is not discussed here.

6.4. Boundary conditions. Enforcing essentially the boundary condition

$$
\mathbf{E} \times\left.\mathbf{n}\right|_{\partial \Omega}=0
$$

is trivial when $\partial \Omega$ is locally a hyperplane which is orthogonal to a Cartesian axis, but this operation becomes a headache when the boundary is arbitrary. This difficulty can be avoided by enforcing the boundary condition naturally. For instance, this is done in [1] by adding Lagrange multipliers. We describe in this section how it can be done by resorting to Nitsche's trick [38.

We replace the family of approximation spaces $\left\{\mathbf{X}_{h}\right\}_{h>0}$ defined in $\$ 2.4$ by a new family $\left\{\mathbf{Y}_{h}\right\}_{h>0}$ conforming in $\mathbf{H}^{1}(\Omega)$, and we assume that there is a family of approximation operators, which we again denote $\left\{\mathcal{C}_{h}\right\}_{h>0}$, so that (2.14)-(2.15) hold for all $\mathbf{F}$ in $\mathbf{H}^{l}(\Omega)$. Given a stabilization parameter $\gamma \geq 0$ large enough and an integer $\chi \in\{0,1\}$, we set

$$
j_{h}(\mathbf{E}, \mathbf{F})=\int_{\partial \Omega}(\nabla \times \mathbf{E}) \cdot(\mathbf{F} \times \mathbf{n})+\chi \int_{\partial \Omega}(\mathbf{E} \times \mathbf{n}) \cdot(\nabla \times \mathbf{F})+\frac{\gamma}{h} \int_{\partial \Omega}(\mathbf{E} \times \mathbf{n}) \cdot(\mathbf{F} \times \mathbf{n}) .
$$

The discrete eigenvalue problem (5.2) is then modified as follows: Seek a triplet $\left(\lambda_{h}, \mathbf{E}_{h}, p_{h}\right) \in \mathbb{R} \times \mathbf{Y}_{h} \backslash\{0\} \times M_{h}$ so that for all $\mathbf{F}_{h} \in \mathbf{Y}_{h}$ and $q_{h} \in M_{h}$,

$$
d_{h}\left(\left(\mathbf{E}_{h}, p_{h}\right),\left(\mathbf{F}_{h}, q_{h}\right)\right)+j_{h}\left(\mathbf{E}_{h}, \mathbf{F}_{h}\right)=\lambda_{h}\left(\mathbf{E}_{h}, \mathbf{F}_{h}\right), \quad \forall\left(\mathbf{F}_{h}, q_{h}\right) \in \mathbf{Y}_{h} \times M_{h}
$$

It can then be shown that all the convergence results of $\$$ remain true provided the discrete norm $\|\cdot\|_{h}$ is modified appropriately. We leave the details of the proofs to the reader.

We have verified numerically that all the results reported in $\$ 6.1$ and $\$ 6.2$ do not depend on the particular technique which is used to enforce the boundary conditions. Whether the boundary conditions are enforced essentially or by using the technique described above, the COC only differ by negligible quantities. We have taken $\gamma=1$ and $\chi=0$ in the numerical simulations. Using $\chi=1$ does not significantly change the results since there is no regularity pick-up on the dual boundary value problem.

\section{ACKNOWLEDGMENT}

The authors are pleased to acknowledge fruitful discussions with Franky Luddens (Univ. Paris XI).

\section{REFERENCES}

[1] F. Assous, P. Degond, E. Heintze, P.-A. Raviart, and J. Segre. On a finite-element method for solving the three-dimensional Maxwell equations. J. Comput. Phys., 109(2):222-237, 1993. MR.1253460 (94j:78003)

[2] I. Babuška and J. Osborn. Eigenvalue problems. In Handbook of numerical analysis, Vol. II, Handb. Numer. Anal., II, pages 641-787. North-Holland, Amsterdam, 1991. MR1115240

[3] D. Boffi, M. Costabel, M. Dauge, and L. Demkowicz. Discrete compactness for the $h p$ version of rectangular edge finite elements. SIAM J. Numer. Anal., 44(3):979-1004, 2006. MR2231852 (2007c:65101)

[4] James H. Bramble and Joseph E. Pasciak. A new approximation technique for div-curl systems. Math. Comp., 73(248):1739-1762 (electronic), 2004. MR2059734(2005b:65124)

[5] James H. Bramble and Xuejun Zhang. The analysis of multigrid methods. In Handbook of numerical analysis, Vol. VII, Handb. Numer. Anal., VII, pages 173-415. North-Holland, Amsterdam, 2000. MR1804746 (2001m:65183) 
[6] J.H. Bramble, T.V. Kolev, and J.E. Pasciak. The approximation of the Maxwell eigenvalue problem using a least-squares method. Math. Comp., 74(252):1575-1598 (electronic), 2005. MR2164087 (2006f:65110)

[7] J.H. Bramble, J.E. Pasciak, and P.S. Vassilevski. Computational scales of Sobolev norms with application to preconditioning. Math. Comp., 69(230):463-480, 2000. MR 1651742 (2000k:65088)

[8] S. C. Brenner, J. Cui, F. Li, and L.-Y. Sung. A nonconforming finite element method for a two-dimensional curl-curl and grad-div problem. Numer. Math., 109(4):509-533, 2008. MR2407321 (2009c:65294)

[9] S.C. Brenner, F. Li, and L.-Y. Sung. Nonconforming Maxwell eigensolvers. J. Sci. Comput., 40(1-3):51-85, 2009. MR2511728 (2010i:65251)

[10] A. Buffa, P. Ciarlet, Jr., and E. Jamelot. Solving electromagnetic eigenvalue problems in polyhedral domains with nodal finite elements. Numer. Math., 113(4):497-518, 2009. MR2545492 (2010i:65252)

[11] A. Buffa, M. Costabel, and M. Dauge. Algbraic convergence for anisotropic edge elements in polyhedral domains. Numer. Math., 101(1):29-65, 2005. MR2194717(2006j:65339)

[12] Annalisa Buffa, Paul Houston, and Ilaria Perugia. Discontinuous Galerkin computation of the Maxwell eigenvalues on simplicial meshes. J. Comput. Appl. Math., 204(2):317-333, 2007. MR2324460 (2008g:65150)

[13] S. Caorsi, P. Fernandes, and M. Raffetto. On the convergence of Galerkin finite element approximations of electromagnetic eigenproblems. SIAM J. Numer. Anal., 38(2):580-607 (electronic), 2000. MR.1770063 (2001e:65172)

[14] Patrick Ciarlet, Jr. and Grace Hechme. Computing electromagnetic eigenmodes with continuous Galerkin approximations. Comput. Methods Appl. Mech. Engrg., 198(2):358-365, 2008. MR2468085 (2009j:78007)

[15] Patrick Ciarlet, Jr., F. Lefèvre, S. Lohrengel, and S. Nicaise. Weighted regularization for composite matherials in electromagnetism. Math. Model. Numer. Anal., 2009. Submitted. MR2647754

[16] Ph. Clément. Approximation by finite element functions using local regularization. Rev. Française Automat. Informat. Recherche Opérationnelle Sér., RAIRO Analyse Numérique, 9(R-2):77-84, 1975. MR0400739 (53:4569)

[17] M. Costabel. A remark on the regularity of solutions of Maxwell's equations on Lipschitz domains. Math. Methods Appl. Sci., 12(4):365-368, 1990. MR1048563 (91c:35028)

[18] M. Costabel. A coercive bilinear form for Maxwell's equations. J. Math. Anal. Appl., 157(2):527-541, 1991. MR.1112332 (92c:35113)

[19] M. Costabel and M. Dauge. Weighted regularization of Maxwell equations in polyhedral domains. A rehabilitation of nodal finite elements. Numer. Math., 93(2):239-277, 2002. MR.1941397 (2004a:78039)

[20] M. Costabel and M. Dauge. Computation of resonance frequencies for Maxwell equations in non-smooth domains. In Topics in computational wave propagation, volume 31 of Lect. Notes Comput. Sci. Eng., pages 125-161. Springer, Berlin, 2003. MR2032869 (2004k:78026)

[21] E. Creusé and S. Nicaise. Discrete compactness for a discontinuous Galerkin approximation of Maxwell's system. M2AN Math. Model. Numer. Anal., 40(2):413-430, 2006. MR2241830 (2008a:65207)

[22] M. Dauge. Elliptic boundary value problems on corner domains, volume 1341 of Lecture Notes in Mathematics. Springer-Verlag, Berlin, 1988. Smoothness and asymptotics of solutions. MR.961439 (91a:35078)

[23] M. Dauge. Benchmark for maxwell. http://perso.univ-rennes1.fr/monique.dauge/ benchmax.html, 2009.

[24] A. Ern and J.-L. Guermond. Theory and practice of finite elements, volume 159 of Applied Mathematical Sciences. Springer-Verlag, New York, 2004. MR2050138 (2005d:65002)

[25] L.C. Evans. Partial differential equations, volume 19 of Graduate Studies in Mathematics. American Mathematical Society, Providence, RI, 1998. MR.1625845 (99e:35001)

[26] D. Gilbarg and N.S. Trudinger. Elliptic partial differential equations of second order. Classics in Mathematics. Springer-Verlag, Berlin, 2001. Reprint of the 1998 edition. MR1814364 (2001k:35004) 
[27] P. Grisvard. Elliptic problems in nonsmooth domains, volume 24 of Monographs and Studies in Mathematics. Pitman (Advanced Publishing Program), Boston, MA, 1985. MR775683 (86m:35044)

[28] C. Hazard and M. Lenoir. On the solution of time-harmonic scattering problems for Maxwell's equations. SIAM J. Math. Anal., 27(6):1597-1630, 1996. MR1416510 (98c:35157)

[29] R. Hiptmair. Finite elements in computational electromagnetism. Acta Numer., 11:237-339, 2002. MR2009375 (2004k:78028)

[30] D. Jerison and C.E. Kenig. The inhomogeneous Dirichlet problem in Lipschitz domains. J. Funct. Anal., 130(1):161-219, 1995. MR.1331981 (96b:35042)

[31] F. Kikuchi. On a discrete compactness property for the Nédélec finite elements. J. Fac. Sci. Univ. Tokyo Sect. IA Math., 36(3):479-490, 1989. MR1039483 (91h:65173)

[32] V. A. Kozlov, V. G. Maz'ya, and J. Rossmann. Spectral problems associated with corner singularities of solutions to elliptic equations, volume 85 of Mathematical Surveys and Monographs. American Mathematical Society, Providence, RI, 2001. MR.1788991 (2001i:35069)

[33] R. B. Lehoucq, D. C. Sorensen, and C. Yang. ARPACK users' guide, volume 6 of Software, Environments, and Tools. Society for Industrial and Applied Mathematics (SIAM), Philadelphia, PA, 1998. Solution of large-scale eigenvalue problems with implicitly restarted Arnoldi methods. MR 1621681

[34] R. Leis. Zur Theorie elektromagnetischer Schwingungen in anisotropen inhomogenen Medien. Math. Z., 106:213-224, 1968. MR0233555 (38:1876)

[35] J.-L. Lions and E. Magenes. Problèmes aux limites non homogènes et applications, volume 1. Dunod, Paris, France, 1968. MR0247243 (40:512)

[36] J.-L. Lions and J. Peetre. Sur une classe d'espaces d'interpolation. Inst. Hautes Études Sci. Publ. Math., 19:5-68, 1964. MR0165343(29:2627)

[37] P. Monk and L. Demkowicz. Discrete compactness and the approximation of Maxwell's equations in $\mathbb{R}^{3}$. Math. Comp., 70(234):507-523, 2001. MR.1709155(2001g:65156)

[38] J. Nitsche. Über ein Variationsprinzip zur Lösung von Dirichlet-Problemen bei Verwendung von Teilräumen, die keinen Randbedingungen unterworfen sind. Abh. Math. Sem. Univ. Hamburg, 36:9-15, 1971. Collection of articles dedicated to Lothar Collatz on his sixtieth birthday. MR 0341903 (49:6649)

[39] J.E. Osborn. Spectral approximation for compact operators. Math. Comp., 29:712-725, 1975. MR0383117(52:3998)

[40] R.L. Scott and S. Zhang. Finite element interpolation of nonsmooth functions satisfying boundary conditions. Math. Comp., 54(190):483-493, 1990. MR.1011446 (90j:65021)

Department of Mathematics, Texas A\&M University, 3368 TAmU, College Station, TEXAs 77843

E-mail address: bonito@math.tamu.edu

Department of Mathematics, Texas A\&M University, 3368 Tamu, College Station, Texas 77843. On Leave from LIMSI, UPR 3251 CNRS, BP 133, 91403 Orsay Cedex, France E-mail address: guermond@math.tamu.edu 